\title{
New Out-of-Plane Angle and Bond Angle Internal Coordinates and Related Potential Energy Functions for Molecular Mechanics and Dynamics Simulations
}

\author{
SANG-HO LEE, KIM PALMO, SAMUEL KRIMM \\ Biophysics Research Division and Department of Physics, The University of Michigan, Ann Arbor, \\ Michigan 48109
}

Received 18 November 1998; accepted 1 March 1999

\begin{abstract}
With currently used definitions of out-of-plane angle and bond angle internal coordinates, Cartesian derivatives have singularities, at $\pm \pi / 2$ in the former case and $\pi$ in the latter. If either of these occur during molecular mechanics or dynamics simulations, the forces are not well defined. To avoid such difficulties, we provide new out-of-plane and bond angle coordinates and associated potential energy functions that inherently avoid these singularities. The application of these coordinates is illustrated by $a b$ initio calculations on ammonia, water, and carbon dioxide. (c) 1999 John Wiley \& Sons, Inc. J Comput Chem 20: 1067-1084, 1999
\end{abstract}

Keywords: internal coordinates; potential energy functions; molecular mechanics; molecular dynamics

\section{Introduction}

A

lthough ab initio quantum mechanical methods can be used for rigorous structural analyses of small molecules, molecular mechanics

Correspondence to: S. Krimm; e-mail: skrimm@umich.edu

Contract/grant sponsor: NSF; contract/grant numbers: MCB-9601006 and DMR-9627786
(MM) or molecular dynamics (MD) simulations are the only effective methods for studying large molecules such as proteins and nucleic acids. An empirical potential energy function for MM or MD simulations is typically expressed in terms of the molecule's internal coordinates (e.g., bond distances, bond angles, out-of-plane angles, torsion angles), while each atom is monitored in Cartesian coordinates. Parameters based on internal coordinates are easily transferable between similar local 
structures, as shown in the development of an $a b$ initio-based spectroscopic force field for $\alpha$-poly(Lalanine). ${ }^{1}$ Similarly, the internal parameters in the potential function can be obtained from those of related small molecules that have similar local molecular structures, such internal parameters being reasonably derived from $a b$ initio calculations or a rigorous experimental analysis based on accurate observed data. However, a problem with some internal coordinates, specifically the out-of-plane angle and the valence angle coordinates, is that their definitions are not unique. As efforts are being made to improve MM functions, ${ }^{2-7}$ it is important to avoid such problems in their formulation. We present here new out-of-plane angle and bond angle internal coordinates, with related new potential energy forms, that improve in many respects on the commonly used ones.

In the Wilson-type internal coordinates ${ }^{8}$ the Cartesian derivatives of an out-of-plane angle coordinate have singularities at $\pm \pi / 2$ and the Cartesian derivatives of a bond angle coordinate have singularities $^{9-11}$ at $\pi$ (local linear structure). If any of these singularities occurs during $\mathrm{MM}$ or $\mathrm{MD}$ simulations, which is not prohibited for any valid physical reason, the Cartesian forces (derivatives of the potential function) are not well defined. Fortunately, the Cartesian derivatives of a torsion angle are well defined except for the case when three related adjacent atoms move into a linear configuration. ${ }^{8,11,12}$ In such a case, the corresponding singularities can be removed by a suitable choice of an angular switching function. ${ }^{13}$

For both the out-of-plane angle and the valence angle internal coordinates we carefully examine the common definitions and present a new coordinate that is well defined in all physically permitted configurations. We also present the corresponding potential energy forms, which at least can satisfy the expected asymptotic behavior and the curvature at the equilibrium conformation. A new linear angle coordinate suitable for a locally linear equilibrium conformation is also given. As applications of these new internal coordinates and their potential energy forms, detailed analyses have been done on the isolated ammonia $\left(\mathrm{NH}_{3}\right)$, water $\left(\mathrm{H}_{2} \mathrm{O}\right)$, and carbon dioxide $\left(\mathrm{CO}_{2}\right)$ molecules.

\section{Theory}

The typical calculation in MM or MD involves the derivatives of the total potential function, $V$, with respect to the Cartesian coordinates of the $\nu$ th atom, $\mathbf{x}_{\nu}^{j}(\nu=1, \ldots, N)$, where $j$ is an index for coordinate components $x, y$, or $z$ :

$$
\begin{aligned}
\frac{\partial V}{\partial \mathbf{x}_{\nu}^{j}}= & \sum_{\beta=1}^{N_{R}} \frac{\partial V}{\partial \mathbf{R}^{\beta}} \frac{\partial \mathbf{R}^{\beta}}{\partial \mathbf{x}_{\nu}^{j}} \\
\frac{\partial^{2} V}{\partial \mathbf{x}_{\mu}^{i} \partial \mathbf{x}_{\nu}^{j}}= & \sum_{\alpha, \beta=1}^{N_{R}} \frac{\partial^{2} V}{\partial \mathbf{R}^{\alpha} \partial \mathbf{R}^{\beta}} \frac{\partial \mathbf{R}^{\alpha}}{\partial \mathbf{x}_{\mu}^{i}} \frac{\partial \mathbf{R}^{\beta}}{\partial \mathbf{x}_{\nu}^{j}} \\
& +\sum_{\beta=1}^{N_{R}} \frac{\partial V}{\partial \mathbf{R}^{\beta}} \frac{\partial^{2} \mathbf{R}^{\beta}}{\partial \mathbf{x}_{\mu}^{i} \partial \mathbf{x}_{\nu}^{j}}
\end{aligned}
$$

where $\mathbf{R}^{\beta}$ is the $\beta$ th internal coordinate of total $N_{R}$, and $\partial \mathbf{R}^{\beta} / \partial \mathbf{x}_{\nu}^{j} \equiv \mathbf{B}_{\nu}^{\beta}$ and $\partial^{2} \mathbf{R}^{\beta} / \partial \mathbf{x}_{\mu}^{i} \partial \mathbf{x}_{\nu}^{j} \equiv$ $\left(\partial / \partial \mathbf{x}_{\mu}^{i}\right)\left(\partial \mathbf{R}^{\beta} / \partial \mathbf{x}_{\nu}^{j}\right) \equiv \mathbf{B}_{\mu^{i}, j}^{\beta^{j}}$ are pure geometrical properties called the first and the second order B-matrix elements, respectively.

Because the internal potential energy of a finite molecule should be invariant under the external translations and rotations, $V$ is a function of interatomic distances $r_{\mu \nu} \equiv\left|\mathbf{x}_{\mu \nu}\right| \equiv\left|\mathbf{x}_{\nu}-\mathbf{x}_{\mu}\right|$, with $\mathbf{x}_{\mu \nu}$ being the distance vector from atom $\mu$ to atom $\nu$. The Cartesian derivatives of $\mathbf{x}_{\mu \nu}$ are found to be

$$
\frac{\partial \mathbf{x}_{\mu \nu}^{j}}{\partial \mathbf{x}_{\lambda}^{k}}=\delta_{j k}\left(\delta_{\lambda \nu}-\delta_{\lambda \mu}\right)
$$

where $\delta_{m n}=0$ for $m \neq n$ and $\delta_{m n}=1$ for $m=n$. Among the $N(N-1) / 2$ distance vectors only $N$ -1 are independent. Similarly, for an internal coordinate $\mathbf{R}^{\beta}$ defined by $L$ atoms, the total numbers of independent first- and second-order B-matrix elements are $L-1$ and $L(L-1) / 2$, respectively. It is convenient to choose the $L-1$ (valence) bond vectors as these independent vectors and to label each of these by $\mathbf{y}_{b} \equiv \mathbf{x}_{\lambda \sigma}(b=1, \ldots, L$ - 1), i.e., to assign a pair of atom indices $(\lambda, \sigma)$ for each bond index $b$. The desired B-matrix elements can be expressed in compact form by

$$
\begin{aligned}
\mathbf{B}_{\nu^{j}}^{\beta} & =\sum_{b=1}^{L-1} \frac{\partial \mathbf{R}^{\beta}}{\partial \mathbf{x}_{\lambda \sigma}^{j}}\left(\delta_{\nu \sigma}-\delta_{\nu \lambda}\right) \equiv \sum_{b=1}^{L-1} \frac{\partial \mathbf{R}^{\beta}}{\partial \mathbf{y}_{b}^{j}} \mathbf{K}_{\nu}^{b} \\
\mathbf{B}_{\mu^{i} \nu} \beta^{j} & =\sum_{b, c=1}^{L-1} \frac{\partial^{2} \mathbf{R}^{\beta}}{\partial \mathbf{x}_{\kappa \omega}^{i} \partial \mathbf{x}_{\lambda \sigma}^{j}}\left(\delta_{\mu \omega}-\delta_{\mu \kappa}\right)\left(\delta_{\nu \sigma}-\delta_{\nu \lambda}\right) \\
& =\sum_{b, c=1}^{L-1} \frac{\partial^{2} \mathbf{R}^{\beta}}{\partial \mathbf{y}_{c}^{i} \partial \mathbf{y}_{b}^{j}} \mathbf{K}_{\mu}^{c} \mathbf{K}_{\nu}^{b}
\end{aligned}
$$

where $\mathbf{K}_{\nu}^{b} \equiv \delta_{\nu \sigma}-\delta_{\nu \lambda}$ for $\mathbf{y}_{b}=\mathbf{x}_{\lambda \sigma}$. Thus, the Cartesian derivatives of $\mathbf{R}^{\beta}$ can be computed from $L-1$ independent derivatives with respect to bond 


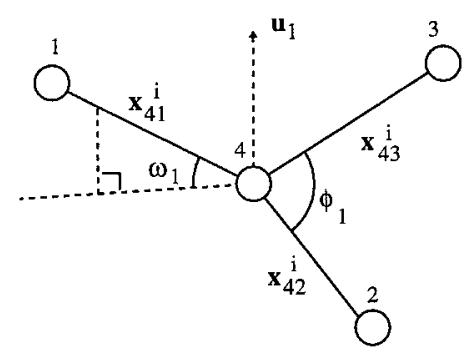

FIGURE 1. Wilson-type out-of-plane coordinate.

vectors $\mathbf{y}_{b} \equiv \mathbf{x}_{\lambda \sigma}$. Because $\mathbf{K}_{\nu}^{b}$ can be only 0 , 1, or -1 , the multiplications involved in eqs. (4) and (5) can be replaced by simple arithmetic summations in a computer program. Each internal coordinate $\mathbf{R}^{\beta}$ defined by $L$ atoms is expressible by a simple form in terms of $L-1$ independent bond vectors, and thus eqs. (4) and (5) can be effective forms for computing B-matrix elements. We believe this formalism is as effective as the method presented by Miller et al., ${ }^{14,15}$ which uses local orthogonal coordinate systems to give simple expressions for nonzero B-matrix elements combined with coordinate transformations based on the translational and rotational invariance in internal coordinates.

\section{Out-of-Plane Angle}

An out-of-plane angle bending coordinate arises when the local molecular structure consists of four atoms with three valence bonds formed to one center atom (see Fig. 1).

The three independent distance vectors can be chosen as the bond vectors $\mathbf{x}_{41} \equiv \mathbf{y}_{1}, \mathbf{x}_{42} \equiv \mathbf{y}_{2}$, and $\mathbf{x}_{43} \equiv \mathbf{y}_{3}$. Let $\phi_{1}$ be the angle between $\mathbf{x}_{42}$ and $\mathbf{x}_{43}$ (the other two angles, $\phi_{2}$ and $\phi_{3}$, can be defined by cyclic permutations among the three bond vectors).

\section{WILSON-TYPE COORDINATE}

A Wilson-type out-of-plane angle coordinate ${ }^{8}$ is defined by an angle, $\omega_{1}$, between the bond vector $\mathbf{x}_{41}$ and the plane containing the other two bond vectors, $\mathbf{x}_{42}$ and $\mathbf{x}_{43}$ :

$$
\sin \omega_{1}=\mathbf{e}_{41} \cdot\left(\frac{\mathbf{e}_{42} \times \mathbf{e}_{43}}{\sin \phi_{1}}\right) \equiv \mathbf{e}_{41} \cdot \mathbf{u}_{1}
$$

where $\mathbf{e}_{41}$ and $\mathbf{u}_{1}$ are unit vectors defined by $\mathbf{e}_{41} \equiv \mathbf{x}_{41} /\left|\mathbf{x}_{41}\right|$ and $\mathbf{u}_{1} \equiv \mathbf{e}_{42} \times \mathbf{e}_{43} / \sin \phi_{1}$, respectively. The corresponding empirical potential func- tion for MM or MD simulations is commonly taken (up to the quadratic term) as

$$
V_{\omega_{1}}=\frac{1}{2} k_{1}\left(\omega_{1}-\omega_{1}^{\mathrm{eq}}\right)^{2}
$$

where $\omega_{1}^{\text {eq }}$ is the value of $\omega_{1}$ at the equilibrium structure and $k_{1}$ is a harmonic force constant parameter related to the out-of-plane angle deformation. In this definition, there are three possible ways to measure the deviation angle from the reference plane. The other two angles, $\omega_{2}$ and $\omega_{3}$, can be defined by cyclic permutations of eq. (6). Rather than a single out-of-plane angle, the symmetrized average of the three is used in usual MM and MD simulations. The first derivatives of $\omega_{1}$ with respect to the related bond vectors are given by

$$
\begin{aligned}
\frac{\partial \omega_{1}}{\partial \mathbf{x}_{41}^{j}} & =\frac{1}{\cos \omega_{1}} \frac{\partial \mathbf{e}_{41}}{\partial \mathbf{x}_{41}^{j}} \cdot \mathbf{u}_{1} \\
\frac{\partial \omega_{1}}{\partial \mathbf{x}_{42}^{j}} & =\frac{1}{\cos \omega_{1}} \mathbf{e}_{41} \cdot \frac{\partial \mathbf{u}_{1}}{\partial \mathbf{x}_{42}^{j}} \\
\frac{\partial \omega_{1}}{\partial \mathbf{x}_{43}^{j}} & =\frac{1}{\cos \omega_{1}} \mathbf{e}_{41} \cdot \frac{\partial \mathbf{u}_{1}}{\partial \mathbf{x}_{43}^{j}} .
\end{aligned}
$$

The first-order B-matrix elements can be obtained by computing $\partial \mathbf{e}_{41} / \partial \mathbf{x}_{41}^{j}, \partial \mathbf{u}_{1} / \partial \mathbf{x}_{42}^{j}$, and $\partial \mathbf{u}_{1} / \partial \mathbf{x}_{43}^{j}$, with $\mathbf{K}_{\nu}^{b}$ elements being given by

$$
\mathbf{K}=\left(\begin{array}{llll}
1 & 0 & 0 & -1 \\
0 & 1 & 0 & -1 \\
0 & 0 & 1 & -1
\end{array}\right)
$$

In most cases, the computation of $\mathbf{B}$-matrix elements involves the derivatives of vectors of type $\mathbf{e}_{41}$ or $\mathbf{u}_{1} \equiv \mathbf{e}_{42} \times \mathbf{e}_{43} / \sin \phi_{1}$ with respect to the related distance vectors, and these can be programmed as subroutines for frequent calling. Expansion to higher order B-matrix elements is straightforward, and the final formula can be expressed in a compact vector notation. The detailed computational methods and expressions for these are given elsewhere. ${ }^{16}$

Note that when the valence angle $\phi_{1}$ is close to $\pi$ or 0 (i.e, $\sin \phi_{1} \approx 0$ ), the expression of eq. (6) cannot be used for numerical computation. Although $\phi_{1}$ cannot take values close to zero due to the van der Waals repulsion between atom 2 and atom 3 , the case of $\phi_{1} \approx \pi$ (linear orientation) may happen during MD simulations. In such a case, a suitable switching function to remove the singularity can be introduced, but the related expressions 
in eqs. (1) and (2) become much more complicated..$^{13}$ Aside from this problem, due to the $\sin \omega_{1}$ term in eq. (6), the Cartesian derivatives of this coordinate, like $\partial \omega_{1} / \partial \mathbf{x}_{\nu}^{j}$ and $\partial^{2} \omega_{1} / \partial \mathbf{x}_{\mu}^{i} \partial \mathbf{x}_{\nu}^{j}$, are not well defined for $\omega_{1}$ close to $\pm \pi / 2$ [see eqs. (8)-(10)], which also may happen during MD simulations. To avoid this kind of singularity at $\omega_{1}=$ $\pm \pi / 2$, sin $\omega_{1}$ itself can be directly used as an out-of-plane coordinate, with the corresponding potential function being expressed in terms of $\sin \omega_{1}$ by

$$
V_{\omega_{1}}=\frac{1}{2} k_{1}\left(\sin \omega_{1}-\sin \omega_{1}^{\mathrm{eq}}\right)^{2}
$$

But the functional form of eq. (12) imposes the result that for a given equilibrium angle $\omega_{1}^{\text {eq }}$ the potential energy at an out-of-plane angle of $\omega_{1}$ is the same as that at $\pi-\omega_{1}$, which is highly unlikely. Also, in general, when two of the three end atoms approach very closely, there is a strong repulsion between them, and the potential energy should go to a very high value. The potential energy forms of eqs. (7) and (12), which are valid only in the neighborhood of the equilibrium conformation, far from correctly describe this expected asymptotic behavior. Even if cubic or quartic anharmonic terms are introduced in eqs. (7) or (12), this problem will still exist.

\section{IMPROPER TORSION COORDINATE}

Another commonly used out-of-plane angle bending coordinate in MM and MD simulations is called an improper torsion, and is defined by a torsion angle $\tau_{1}(3-2-1-4)$ with two nonbonded distance vectors $\mathbf{x}_{23}$ and $\mathbf{x}_{21}$ (the explicit definition of a torsion angle $\tau(\lambda-\mu-\nu-\sigma)$ with atom indices and related derivatives with respect to bond vectors are shown in the appendix). The other two associated improper torsions are $\tau_{2}(1-3-2-4)$ and $\tau_{3}(2-1-3-4)$, respectively. The symmetrized average of $\tau_{1}, \tau_{2}$, and $\tau_{3}$ is commonly used as a representative out-of-plane coordinate, with a quadratic potential energy form similar to eq. (7) for each improper torsion coordinate. Although this kind of improper torsion coordinate can avoid the singularity problem at $\omega_{1}=$ $\pm \pi / 2$ inherent in a coordinate defined by eq. (6), the frequently overlooked fact is that this kind of coordinate depends on the valence bond lengths $r_{41}, r_{42}$, and $r_{43}$ for nonplanar configurations. From the derivative of a unit vector $\mathbf{e}_{\lambda \nu}=\mathbf{x}_{\lambda \nu} / r_{\lambda \nu}$ with respect to the length $r_{\lambda \nu}(\neq 0)$ we obtain

$$
\frac{\partial \mathbf{x}_{\lambda \nu}}{\partial r_{\lambda \mu}}=\mathbf{e}_{\lambda \nu} .
$$

Applying this to the results from the appendix with the chain rule, the derivatives of $\tau_{1}$ with respect to bond distances are found to be

$$
\begin{aligned}
& \frac{\partial \tau_{1}}{\partial r_{41}}=\frac{\cos \theta_{2}}{r_{21} \sin \theta_{2}} \mathbf{u} \cdot \mathbf{e}_{41} \\
& \frac{\partial \tau_{1}}{\partial r_{42}}=\frac{r_{21}-r_{23} \cos \theta_{2}}{r_{21} r_{23} \sin \theta_{2}} \mathbf{u} \cdot \mathbf{e}_{42} \\
& \frac{\partial \tau_{1}}{\partial r_{43}}=-\frac{1}{r_{23} \sin \theta_{2}} \mathbf{u} \cdot \mathbf{e}_{43}
\end{aligned}
$$

where $\mathbf{u}$ is a unit vector defined by $\mathbf{u} \equiv \mathbf{e}_{21} \times$ $\mathbf{e}_{23} / \sin \theta_{2}$, which is perpendicular to the plane of the three end atoms 1,2 , and 3, with $\theta_{2}$ being the angle between $\mathbf{x}_{21}$ and $\mathbf{x}_{23}$. Note that for any nonplanar structure $\mathbf{u}$ is not perpendicular to the unit vectors $\mathbf{e}_{41}, \mathbf{e}_{42}$, and $\mathbf{e}_{43}$, giving a nonzero derivative with respect to each bond length internal coordinate. In such an interdependent internal coordinate set it is difficult to get consistent internal parameters that can be transferable between similar local structures. In a recent report by Maple et al. $^{4}$ it is mentioned that a symmetrized Wilson-type out-of-plane angle was found to give a much better description of the potential energy surface than the conventional improper torsion angle.

Instead of the conventional improper torsion angle, let us consider the torsion angle $\tau_{1}(3-2-$ $4-1)\left(\tau_{1}^{\mathrm{eq}}=\pi\right.$ for a planar equilibrium structure), which has only one nonbonded distance vector $\mathbf{x}_{23}$. Similarly, the other two associated improper torsions can be chosen as $\tau_{2}(1-3-4-2)$ and $\tau_{3}(2$ $-1-4-3)$, respectively. In the case of $\tau_{1}$, the unit bond vectors $\mathbf{e}_{42}$ and $\mathbf{e}_{43}$ are perpendicular to $\mathbf{e}_{24} \times \mathbf{e}_{23}$ and the unit bond vectors $\mathbf{e}_{41}$ and $\mathbf{e}_{42}$ are perpendicular to $\mathbf{e}_{41} \times \mathbf{e}_{42}$. Thus, the derivatives of $\tau_{1}$ with respect to $r_{41}, r_{42}$, and $r_{43}$ are all zero, and the above bond-length dependence disappears in this type of definition.

\section{A NEW OUT-OF-PLANE ANGLE COORDINATE}

To introduce a more general out-of-plane coordinate, which is independent of internal bond lengths and is well defined in all physically possible configurations, let us consider a pyramidal structure constructed from three unit bond vectors 


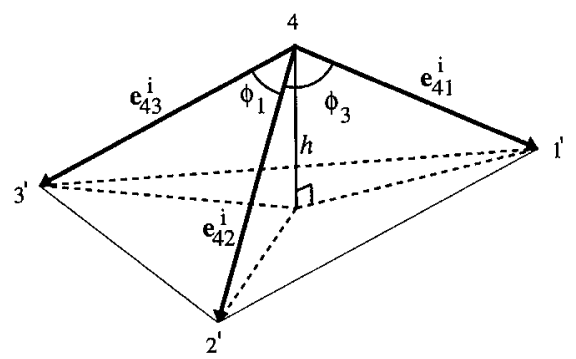

FIGURE 2. New out-of-plane coordinate, $h(\equiv \sin \gamma, \gamma$ $=$ the corresponding out-of-plane angle).

$\mathbf{e}_{41}, \mathbf{e}_{42}$, and $\mathbf{e}_{43}$, as shown in Figure 2. A vector $\mathbf{v}$, which is perpendicular to the plane defined by the three end points of $\mathbf{e}_{41}, \mathbf{e}_{42}$, and $\mathbf{e}_{43}$, can be defined by

$$
\mathbf{v} \equiv \mathbf{e}_{41} \times \mathbf{e}_{42}+\mathbf{e}_{42} \times \mathbf{e}_{43}+\mathbf{e}_{43} \times \mathbf{e}_{41} .
$$

The unit vector $\hat{\mathbf{v}}=(\mathbf{v} / v)$ in the direction of $\mathbf{v}$ can be obtained from

$$
\begin{aligned}
v^{2}= & 3-\cos ^{2} \phi_{1}-\cos ^{2} \phi_{2}-\cos ^{2} \phi_{3}+ \\
& +2\left\{\cos \phi_{1}\left(\cos \phi_{2}-1\right)+\cos \phi_{2}\left(\cos \phi_{3}-1\right)\right. \\
& \left.+\cos \phi_{3}\left(\cos \phi_{1}-1\right)\right\} .
\end{aligned}
$$

We define the desired new coordinate as the normal distance, $h$, from the apex atom 4 to the point in the plane determined by the three end points of $\mathbf{e}_{41}, \mathbf{e}_{42}$, and $\mathbf{e}_{43}$. Explicitly, $h$ is found to be

$$
h=\hat{\mathbf{v}} \cdot \mathbf{e}_{41}=\hat{\mathbf{v}} \cdot \mathbf{e}_{42}=\hat{\mathbf{v}} \cdot \mathbf{e}_{43}=\frac{1}{v} \mathbf{e}_{41} \cdot\left(\mathbf{e}_{42} \times \mathbf{e}_{43}\right),
$$

which is a dimensionless quantity with 1 as its maximum value. From eq. (19) it is evident that this new coordinate, $h$, is ill-defined at $v=0$, viz., when two of the three end atoms occupy the same point. But this can never happen in real situations due to the van der Waals repulsion between such atoms. Although a symmetrized form of the three related improper torsions, or Wilson-type coordinates, is commonly used in MM and MD simulations, such a symmetrization is not necessary in the new coordinate, because $h$ itself is in a symmetric form and well represents the deviation from planarity. This new coordinate is essentially different from that in the recent treatment by Tuzun et $\mathrm{al}^{17}$ Instead of using unit bond vectors, they used the three bond vectors $\mathbf{x}_{41}, \mathbf{x}_{42}$, and $\mathbf{x}_{43}$ to separate three out-of-plane angles from three in- plane angles by projection to the plane defined by three end atoms 1,2, and 3. Those out-of-plane deformations depend on the internal bond lengths for nonplanar orientations, although they are orthogonal to the three in-plane angle bends.

The first derivatives of $h$ with respect to bond vectors are found to be

$$
\begin{gathered}
\frac{\partial h}{\partial \mathbf{x}_{41}}=\frac{1}{v}\left(-h \frac{\partial v}{\partial \mathbf{x}_{41}}+\frac{\partial \mathbf{e}_{41}}{\partial \mathbf{x}_{41}} \cdot\left(\mathbf{e}_{42} \times \mathbf{e}_{43}\right)\right) \\
\frac{\partial h}{\partial \mathbf{x}_{42}}=\frac{1}{v}\left(-h \frac{\partial v}{\partial \mathbf{x}_{42}}+\mathbf{e}_{41} \cdot\left(\frac{\partial \mathbf{e}_{42}}{\partial \mathbf{x}_{42}} \times \mathbf{e}_{43}\right)\right) \\
\frac{\partial h}{\partial \mathbf{x}_{43}}=\frac{1}{v}\left(-h \frac{\partial v}{\partial \mathbf{x}_{43}}+\mathbf{e}_{41} \cdot\left(\mathbf{e}_{42} \times \frac{\partial \mathbf{e}_{43}}{\partial \mathbf{x}_{43}}\right)\right)
\end{gathered}
$$

where $\partial v / \partial \mathbf{x}_{4 j}$ can be obtained from eq. (18) whenever we compute the derivatives of type $\partial \mathbf{e}_{4 j} / \partial \mathbf{x}_{4 j}(j=1,2,3)$. Generalization to a higher order computation is straightforward.

A suitable potential energy function for our out-of-plane deformation should have the properties that its energy goes to infinity as the three end atoms converge to one point $(h \rightarrow 1)$ and the state of planar orientation $(h=0)$ is a transition state with an energy higher than that of the equilibrium nonplanar conformation (the difference between the two is called the barrier height, $B_{h}$ ). A simple functional form that satisfies these requirements, which is symmetric in terms of $h$, is for a nonplanar equilibrium structure

$$
\begin{array}{r}
V_{h}=V_{2}\left(\frac{|h|^{t}}{1-|h|^{s}}\right)^{2}+V_{4}\left(\frac{|h|^{t}}{1-|h|^{s}}\right)^{4}, \\
(-1<h<1)
\end{array}
$$

where $s, t, V_{2}$, and $V_{4}$ are parameters to be fit. (The constant energy term, $V_{0}$, can be put to zero by an energy scaling. For a planar equilibrium structure, we can simply take the quadratic term (i.e., $V_{4}=0$ ).) In most cases, the actual value of $t$, which can be a noninteger, should be close to 1 , and when the value of $s$ (positive integer) becomes large, the potential function behaves like a simple polynomial of $h^{2}$ in the neighborhood of $h=0$. When $s$ is an even number, the absolute signs in eq. (23) can be left out, giving a simpler potential form. At an equilibrium configuration, we have the following relations:

$$
V_{2}=-2 V_{4}\left(\frac{\left|h^{\mathrm{eq}}\right|^{t}}{1-\left|h^{\mathrm{eq}}\right|^{s}}\right)^{2}
$$




$$
\begin{gathered}
\left.f_{\mathrm{hh}} \equiv \frac{\partial^{2} V_{h}}{\partial h^{2}}\right|_{h=h^{\mathrm{eq}}} \\
=8 V_{4}\left(\frac{\left|h^{\mathrm{eq}}\right|^{t}}{1-\left|h^{\mathrm{eq}}\right|^{s}}\right)^{2}\left\{\frac{\left|h^{\mathrm{eq}}\right|^{t}\left[t+(s-t)\left|h^{\mathrm{eq}}\right|^{s}\right]}{\left|h^{\mathrm{eq}}\right|\left(1-\left|h^{\mathrm{eq}}\right|^{s}\right)^{2}}\right\}^{2} \\
B_{h}=-V_{h}^{\mathrm{eq}}=V_{4}\left(\frac{\left|h^{\mathrm{eq}}\right|^{t}}{1-\left|h^{\mathrm{eq}}\right|^{s}}\right)^{4} \\
=\frac{1}{8} f_{\mathrm{hh}}\left\{\frac{\left|h^{\mathrm{eq}}\right|\left(1-\left|h^{\mathrm{eq}}\right|^{s}\right)}{t+(s-t)\left|h^{\mathrm{eq}}\right|^{s}}\right\}^{2}
\end{gathered}
$$

The equilibrium position $h^{\text {eq }}$, the curvature $f_{\text {hh }}$, and the barrier height $B_{h}$, can be estimated from $a b$ initio quantum mechanical calculations for a small molecule. Using the $a b$ initio estimates of $h^{\text {eq }}$ and $f_{\mathrm{hh}}$, the $s$ and $t$ can be chosen to give the $a b$ initio $B_{h}$ and potential energy surface. We illustrate below the application of this new potential function with the new out-of-plane coordinate by a detailed analysis of an isolated $\mathrm{NH}_{3}$ molecule.

\section{Bond Angle}

A conventional internal bond angle coordinate is defined as the angle between two related bonds connected at a center atom, as in Figure 3: the related bond angle, $\phi_{2}$, can be obtained from

$$
\cos \phi_{2}=\mathbf{e}_{21} \cdot \mathbf{e}_{23} .
$$

The first derivatives of $\phi_{2}$ with respect to the related bond vectors are found to be

$$
\begin{aligned}
& \frac{\partial \phi_{2}}{\partial \mathbf{x}_{21}}=-\frac{1}{\sin \phi_{2}} \frac{\partial \mathbf{e}_{21}}{\partial \mathbf{x}_{21}} \cdot \mathbf{e}_{23} \\
& \frac{\partial \phi_{2}}{\partial \mathbf{x}_{23}}=-\frac{1}{\sin \phi_{2}} \mathbf{e}_{21} \cdot \frac{\partial \mathbf{e}_{23}}{\partial \mathbf{x}_{23}} .
\end{aligned}
$$

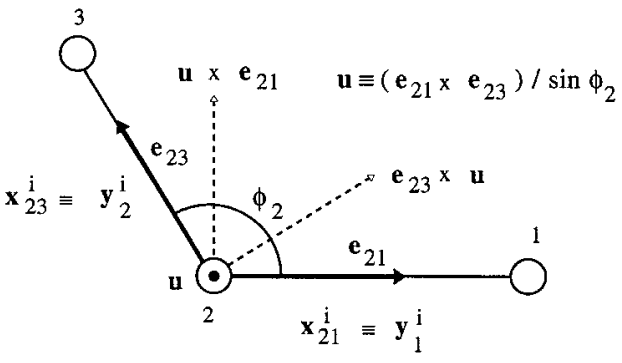

FIGURE 3. Nonlinear angle coordinate.
The related B-matrix elements can be obtained from eq. (4), with $\mathbf{K}_{\nu}^{b}$ elements being given by

$$
\mathbf{K}=\left(\begin{array}{lll}
1 & -1 & 0 \\
0 & -1 & 1
\end{array}\right)
$$

The expansion to a higher order is straightforward, with only derivatives of each unit bond vector with respect to its bond vector required to be computed. The related potential energy form for $\mathrm{MM}$ and $\mathrm{MD}$ simulations is commonly taken (up to the quadratic term) to be

$$
V_{\phi}=\frac{1}{2} k_{\phi}\left(\phi_{2}-\phi_{2}^{\mathrm{eq}}\right)^{2}
$$

where $k_{\phi}$ is a harmonic force constant parameter. Because Cartesian derivatives of $\phi_{2}$ have singularities at $\phi_{2}=0$ and $\phi_{2}=\pi$ [see eqs. (28) and (29)], the Cartesian derivatives of eqs. (1) and (2) are not well defined at these molecular orientations. Although the case of $\phi_{2}=0$ cannot happen due to van der Waals repulsion, that of $\phi_{2}=\pi$ (linear orientation) may happen in MM or MD simulations. To avoid this problem, instead of using $\phi_{2}$, the direct use of $\cos \phi_{2}$ as the related bond angle coordinate was suggested with $V_{\phi}$ being given by ${ }^{9}$

$$
V_{\phi}=\frac{1}{2} k_{\phi}\left(\cos \phi_{2}-\cos \phi_{2}^{\mathrm{eq}}\right)^{2} \text {. }
$$

A desired potential function for a bond-angle deformation should have symmetric double minima about the linear transition configuration (a single minimum for a linear equilibrium conformation), with its value approaching infinity as the distance between the two end atoms converges to zero $\left(\phi_{2} \rightarrow 0\right){ }^{18}$ The form of eq. (32), even if cubic or quartic anharmonic terms are added, is inadequate to reproduce such expected barrier height and asymptotic behavior.

As a new internal bond-angle coordinate, we introduce the magnitude of the dimensionless vector, $\mathrm{g}$, defined by

$$
\mathbf{g} \equiv \frac{1}{2}\left(\mathbf{e}_{21}-\mathbf{e}_{23}\right),
$$

i.e., $g \equiv|\mathbf{g}|$ (the half distance between the two end points of $\mathbf{e}_{21}$ and $\mathbf{e}_{23}$ ), given by

$$
g^{2}=\frac{1}{2}\left(1-\mathbf{e}_{21} \cdot \mathbf{e}_{23}\right)=\left[\sin \left(\phi_{2} / 2\right)\right]^{2} .
$$

The corresponding B-matrix elements can be obtained from the derivatives with respect to re- 
lated bond vectors:

$$
\begin{aligned}
& \frac{\partial g}{\partial \mathbf{x}_{21}^{j}}=-\frac{1}{4 g} \frac{\partial \mathbf{e}_{21}}{\partial \mathbf{x}_{21}^{j}} \cdot \mathbf{e}_{23} \\
& \frac{\partial g}{\partial \mathbf{x}_{23}^{j}}=-\frac{1}{4 g} \mathbf{e}_{21} \cdot \frac{\partial \mathbf{e}_{23}}{\partial \mathbf{x}_{23}^{j}}
\end{aligned}
$$

$$
\begin{aligned}
& \frac{\partial^{2} g}{\partial \mathbf{x}_{21}^{i} \partial \mathbf{x}_{21}^{j}} \\
& \quad=-\frac{1}{4 g}\left(4 \frac{\partial g}{\partial \mathbf{x}_{21}^{i}} \frac{\partial g}{\partial \mathbf{x}_{21}^{i}}+\frac{\partial^{2} \mathbf{e}_{21}}{\partial \mathbf{x}_{21}^{i} \partial \mathbf{x}_{21}^{j}} \cdot \mathbf{e}_{23}\right)
\end{aligned}
$$

$$
\begin{aligned}
& \frac{\partial^{2} g}{\partial \mathbf{x}_{23}^{i} \partial \mathbf{x}_{23}^{j}} \\
& \quad=-\frac{1}{4 g}\left(4 \frac{\partial g}{\partial \mathbf{x}_{23}^{i}} \frac{\partial g}{\partial \mathbf{x}_{23}^{j}}+\mathbf{e}_{21} \cdot \frac{\partial^{2} \mathbf{e}_{23}}{\partial \mathbf{x}_{23}^{i} \partial \mathbf{x}_{23}^{j}}\right)
\end{aligned}
$$

$$
\frac{\partial^{2} g}{\partial \mathbf{x}_{23}^{i} \partial \mathbf{x}_{21}^{j}}=-\frac{1}{4 g}\left(4 \frac{\partial g}{\partial \mathbf{x}_{23}^{i}} \frac{\partial g}{\partial \mathbf{x}_{21}^{j}}+\frac{\partial \mathbf{e}_{21}}{\partial \mathbf{x}_{21}^{j}} \cdot \frac{\partial \mathbf{e}_{23}}{\partial \mathbf{x}_{23}^{i}}\right) \text {. }
$$

Thus, the Cartesian derivatives of $g$ are well defined except for the case of $g=0\left(\phi_{2}=0\right)$, which never happens.

A simple and appropriate potential energy form in this coordinate, which is symmetric about the axis of $g=1$, is given by

$$
\begin{aligned}
V_{g}=V_{1}\left(\frac{|1-g|^{t}}{1-|1-g|^{s}}\right)+V_{2} & \left(\frac{|1-g|^{t}}{1-|1-g|^{s}}\right)^{2}, \\
& (0<g<2) .
\end{aligned}
$$

This looks like a shifted form of the potential function for the out-of-plane coordinate of eq. (23), with the right-hand side shifted by unity and with a different power of each term due to the nonlinear relation of eq. (34). Although the actual value $g$ can take is only from 0 to 1 [the absolute signs in eq. (40) can be neglected in real computations], for a particular configuration of $g=g_{0}\left(\phi_{2}=\phi_{2}^{0}\right)$ we consider the inverted configuration as the state of $g=1+g_{0}\left(\phi_{2}=-\phi_{2}^{0}\right.$ or $\left.\pi+\phi_{2}^{0}\right)$. The related parameters can be determined from relations at the equilibrium conformation:

$$
V_{1}=-2 V_{2}\left(\frac{\left|1-g^{\mathrm{eq}}\right|^{t}}{1-\left|1-g^{\mathrm{eq}}\right|^{s}}\right)
$$

$$
\begin{aligned}
f_{\mathrm{gg}} & \left.\equiv \frac{\partial^{2} V_{g}}{\partial g^{2}}\right|_{g=g^{\mathrm{eq}}} \\
& =2 V_{2}\left\{\frac{\left|1-g^{\mathrm{eq}}\right|^{t-1}\left[t+(s-t)\left|1-g^{\mathrm{eq}}\right|^{s}\right]}{\left(1-\left|1-g^{\mathrm{eq}}\right|^{s}\right)^{2}}\right\}^{2} \\
B_{h} & =V_{2}\left(\frac{\left|1-g^{\mathrm{eq}}\right|^{t}}{1-\left|1-g^{\mathrm{eq}}\right|^{s}}\right)^{2} \\
& =\frac{1}{2} f_{\mathrm{gg}}\left\{\frac{\left|1-g^{\mathrm{eq}}\right|\left(1-\left|1-g^{\mathrm{eq}}\right|^{s}\right)}{t+(s-t)\left|1-g^{\mathrm{eq}}\right|^{s}}\right\}^{2}
\end{aligned}
$$

Due to the inherent double minima nature in an angle bend potential function, it is natural to consider a tunneling effect and a large amplitude inversion motion across the barrier when $B_{h}$ is not large compared to the fundamental frequency of the corresponding angle bend mode. In such a case, each energy level of the harmonic bending mode is expected to split into two levels as in the case of an isolated ammonia molecule, ${ }^{19}$ and this effect should be taken into account in the related spectroscopic band analysis. We illustrate below the application of this new bond angle coordinate and the related potential form by a detailed analysis of an isolated $\mathrm{H}_{2} \mathrm{O}$ molecule.

As shown above, the new bond angle coordinate, $g$, has well-defined Cartesian derivatives for all physically possible values of $g$. Thus, it can always be used in MM and MD calculations. However, in spectroscopic force-field calculations a linear structure poses a problem. This is due to the fact that at a linear orientation $\left(\phi_{2}=\pi, g=1\right)$, the two vectors $\partial \mathbf{e}_{21} / \partial \mathbf{x}_{21}^{k}$ and $\partial \mathbf{e}_{23} / \partial \mathbf{x}_{23}^{k}$ in eqs. (35) and (36) are perpendicular to $\mathbf{e}_{21}$ and $\mathbf{e}_{23}$, giving zero B-matrix elements for $g$ (and thus giving zero vibrational frequency for the corresponding mode). In this case, there is an additional (linear angle bend) degree of freedom in the direction perpendicular to the corresponding linear axis. For a proper description of the linear bending motion it is, therefore, necessary to introduce an arbitrary reference point, $\mathbf{x}_{4}$, to fix the two arbitrary axes perpendicular to the linear axis (Fig. 4). The desired orthonormal basis vectors can be chosen as $\left\{\mathbf{e}_{23}, \mathbf{u}, \mathbf{w}\right\}$ with $\mathbf{u}$ and $\mathbf{w}$ being defined, respectively, by

$$
\begin{aligned}
\mathbf{u} & \equiv \frac{\mathbf{e}_{23} \times \mathbf{e}_{24}}{\sin \phi} \\
\mathbf{w} & \equiv \mathbf{u} \times \mathbf{e}_{23}
\end{aligned}
$$




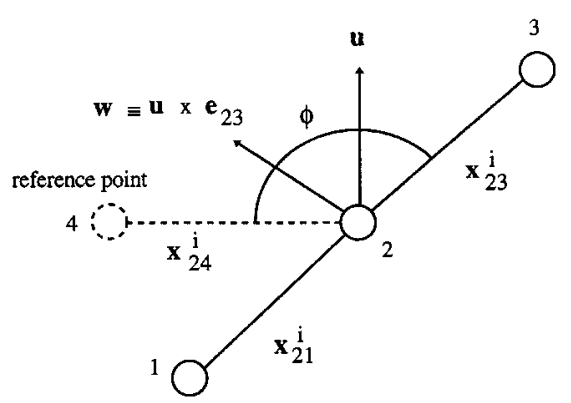

FIGURE 4. Linear angle coordinate.

where $\phi$ is the angle between $\mathbf{e}_{23}$ and $\mathbf{e}_{24}$. The conventional linear angle displacements, $\Delta \gamma_{u}$ and $\Delta \gamma_{w}$, can be obtained ${ }^{20}$ from the derivatives of

$$
\begin{aligned}
\sin \gamma_{u} & \equiv \mathbf{u} \cdot\left(\mathbf{e}_{21} \times \mathbf{e}_{23}\right) \\
\sin \gamma_{w} & \equiv \mathbf{w} \cdot\left(\mathbf{e}_{21} \times \mathbf{e}_{23}\right)=\mathbf{u} \cdot \mathbf{e}_{21} .
\end{aligned}
$$

Considering that a torsion angle displacement is well defined at $\tau= \pm \pi$, new linear angle coordinates can be defined by two torsion angles, $\theta_{u}=$ $\tau(1-u-2-3)$ and $\theta_{w}=\tau(1-w-2-3)$, where $u$ and $w$ represent the position vectors, $\mathbf{x}_{u} \equiv \mathbf{x}_{2}+\mathbf{u}$ and $\mathbf{x}_{w} \equiv \mathbf{x}_{2}+\mathbf{w}$, respectively. Because $\mathbf{e}_{23}$ is orthogonal to $\mathbf{u}$ and $\mathbf{w}$, using the results from the appendix, the first derivatives with respect to related bond vectors are found to be

$$
\begin{aligned}
\frac{\partial \theta_{u}}{\partial \mathbf{x}_{u 1}^{j}} & =-\frac{1}{r_{u 1} \sin \phi_{u}} \hat{\mathbf{p}}^{j} \\
\frac{\partial \theta_{u}}{\partial \mathbf{x}_{2 u}^{j}} & =0 \\
\frac{\partial \theta_{u}}{\partial \mathbf{x}_{23}^{j}} & =\frac{1}{r_{23}} \hat{\mathbf{q}}^{j}
\end{aligned}
$$

where $\phi_{u}$ is the angle between $\mathbf{x}_{u 2}$ and $\mathbf{x}_{u 1}$ with $\hat{\mathbf{p}} \equiv\left(\mathbf{x}_{u 2} \times \mathbf{x}_{u 1}\right) /\left|\mathbf{x}_{u 2} \times \mathbf{x}_{u 1}\right|$ and $\hat{\mathbf{q}} \equiv \mathbf{e}_{23} \times \mathbf{u}$. Because $\cos \phi_{u}=1 / r_{u 1}$, we have

$$
\frac{\partial \theta_{u}}{\partial \mathbf{x}_{u 2}^{j}}=-\frac{\partial \theta_{u}}{\partial \mathbf{x}_{u 1}^{j}} .
$$

Using eqs. (49) and (51), the derivatives for the second-order B-matrix elements are found to be

$$
\begin{aligned}
\frac{\partial^{2} \theta_{u}}{\partial \mathbf{x}_{u 1}^{i} \partial \mathbf{x}_{u 1}^{j}}= & -\frac{1}{\left(r_{u 1} \sin \phi_{u}\right)^{2}} \\
& \times\left\{2(\hat{\mathbf{p}} \times \mathbf{u})^{i} \hat{\mathbf{p}}^{j}+(\hat{\mathbf{i}} \times \mathbf{u})^{j}\right\}
\end{aligned}
$$

$$
\begin{aligned}
& \frac{\partial^{2} \theta_{u}}{\partial \mathbf{x}_{23}^{i} \partial \mathbf{x}_{23}^{j}}=\frac{1}{\left(r_{23}\right)^{2}}\left\{2(\hat{\mathbf{q}} \times \mathbf{u})^{i} \hat{\mathbf{q}}^{j}+(\hat{\mathbf{i}} \times \mathbf{u})^{j}\right\} \\
& \frac{\partial^{2} \theta_{u}}{\partial \mathbf{x}_{u 2}^{i} \partial \mathbf{x}_{u 2}^{j}}=\frac{\partial^{2} \theta_{u}}{\partial \mathbf{x}_{u 1}^{i} \partial \mathbf{x}_{u 1}^{j}}=-\frac{\partial^{2} \theta_{u}}{\partial \mathbf{x}_{u 2}^{i} \partial \mathbf{x}_{u 1}^{j}}
\end{aligned}
$$

where a unit vector $\hat{\mathbf{i}}$ is defined as $\hat{\mathbf{1}} \equiv(1,0,0)$, $\hat{\mathbf{2}} \equiv(0,1,0)$, and $\hat{\mathbf{3}}=(0,0,1)$. (The corresponding formulas for $\theta_{w}$ can be easily obtained by replacing $\mathbf{u}$ by $\mathbf{w}$ with suitable modifications.) The B-matrix elements can be obtained from eqs. (4)-(5), with $\mathbf{K}_{\nu}^{b}$ elements being given by

$$
\mathbf{K}=\left(\begin{array}{rrr}
1 & 0 & 0 \\
0 & 1 & 0 \\
0 & -1 & 0 \\
0 & -1 & 1
\end{array}\right)
$$

where the row distance vector index corresponds to the order of $\mathbf{x}_{u 1}, \mathbf{x}_{u 2}, \mathbf{x}_{2 u}$, and $\mathbf{x}_{23}$. Eqs. (49) and (51) insure that, in this new linear angle coordinate definition, the computation of $\mathbf{B}$-matrix elements is simpler and faster than in the usual definition of eqs. (46) and (47). Note that eqs. (48)-(54) are also well defined for any nonlinear bond-angle structure and either $\theta_{u}$ or $\theta_{w}$ (specifically, $\theta_{u}$ for a choice of the reference point in the plane of atoms 1,2 , and 3) can be used for a nonlinear bond angle coordinate with a suitable choice of the reference point.

For a linear equilibrium structure, the two linear bending modes corresponding to $\theta_{u}$ and $\theta_{w}$ are degenerate, and the related potential functions should be expressed in the same form. Rather than constructing a potential function in terms of $\theta_{u}$ or $\theta_{w}$, let us consider the potential energy form of eq. (40) with $t=1, V_{1}>0$, and $V_{2}>0$. From the nonlinear relation of eq. (34), $V_{1}$ can be estimated by

$$
\begin{aligned}
{\left[\frac{\partial^{2} V_{g}}{\partial \theta_{u} \partial \theta_{u}}\right]_{\theta_{u}=\pi} } & =\left[\frac{\partial^{2} V_{g}}{\partial \theta_{w} \partial \theta_{w}}\right]_{\theta_{w w}=\pi} \\
& =-\frac{1}{4}\left[\frac{\partial V_{g}}{\partial g}\right]_{g=1}=\frac{1}{4} V_{1}
\end{aligned}
$$

where the derivative of $\partial V_{g} / \partial g$ is not continuous at $g=1$, and only its left-hand side limiting value has to be taken because the real range of $g$ is $0<g \leq 1$. Note that in a linear equilibrium conformation we have $\left[\partial V_{g} / \partial \mathbf{x}_{\nu}^{j}\right]_{g=1}=0$ and $\left[\partial^{2} V_{g} / \partial \mathbf{x}_{\nu}^{j} \partial \mathbf{x}_{\nu}^{j}\right]_{g=1} \geq 0$, because the first-order B-matrix elements of $g$ are all zero, and $g$ has a 
maximum value of unity there, respectively [see eqs. (1)-(2)], satisfying the desired equilibrium conditions. Therefore, the potential energy form of eq. (40) with $t=1, V_{1}>0$, and $V_{2}>0$ is also well suited for $\mathrm{MM}$ and $\mathrm{MD}$ simulations in a linear equilibrium structure by using the new angle bend coordinate, $g$. We show below that a calculated $a b$ initio potential energy surface of an isolated $\mathrm{CO}_{2}$ molecule is well reproduced by this model potential energy function.

\section{Redundancy Relations}

When some of the internal coordinates, say $\left\{\mathbf{q}_{j} \mid \mathbf{q}_{j}=\mathbf{R}^{\beta_{j}}, j=1, \ldots, m \leq N_{R}\right\}$, are connected through a relation

$$
\Phi\left(\mathbf{q}_{1}, \mathbf{q}_{2}, \ldots, \mathbf{q}_{m}\right)=0
$$

the related quadratic force constants are not uniquely determined in this dependent coordinate set. To solve this problem, the redundancy function $\Phi$ is usually expanded in a Taylor series as

$$
\begin{aligned}
\Phi(\mathbf{q})= & \sum_{j=1}^{m} \Delta \mathbf{q}_{j}\left[\frac{\partial \Phi}{\partial \mathbf{q}_{j}}\right]_{\mathbf{q}=\mathbf{q}^{e c}} \\
& +\sum_{i, j=1}^{m} \frac{1}{2} \Delta \mathbf{q}_{i} \Delta \mathbf{q}_{j}\left[\frac{\partial^{2} \Phi}{\partial \mathbf{q}_{i} \partial \mathbf{q}_{j}}\right]_{\mathbf{q}=\mathbf{q}^{e c}}+\cdots
\end{aligned}
$$

with $\mathbf{q} \equiv\left(\mathbf{q}_{1}, \mathbf{q}_{2}, \ldots, \mathbf{q}_{m}\right)$ and $\Delta \mathbf{q} \equiv \mathbf{q}-\mathbf{q}^{\text {ec }}$ where $\mathbf{q}^{\mathrm{ec}}$ is an arbitrary expansion center (which is the equilibrium conformation in a spectroscopic formalism) on the hypersurface defined by eq. (57) giving $\Phi\left(\mathbf{q}^{\text {ec }}\right)=0$. The nonlinear terms, $\left[\partial^{2} \Phi / \partial \mathbf{q}_{i} \partial \mathbf{q}_{j}\right]_{\mathbf{q}=\mathbf{q}^{\text {ec }}}$, of eq. (58) usually affect the related harmonic force constants in spectroscopic formalisms. ${ }^{20,21}$ However, in MM and MD formalisms they do not make the quadratic force constants indeterminate. ${ }^{22}$

For a planar trigonal system, the set of conventional bond-angle coordinates has a linear redundancy relation: ${ }^{8}$

$$
\phi_{1}+\phi_{2}+\phi_{3}=2 \pi
$$

where $\phi_{1}, \phi_{2}$, and $\phi_{3}$ are angles between the related bonds (see Fig. 1). In terms of the new angle bend coordinate of eq. (34), from $\sin \left(\phi_{1} / 2+\right.$ $\left.\phi_{2} / 2+\phi_{3} / 2\right)=0$ we have a nonlinear redun- dancy relation:

$$
\begin{aligned}
\Phi= & g_{1} \cos \frac{\phi_{2}}{2} \cos \frac{\phi_{3}}{2}+g_{2} \cos \frac{\phi_{3}}{2} \cos \frac{\phi_{1}}{2} \\
& +g_{3} \cos \frac{\phi_{1}}{2} \cos \frac{\phi_{2}}{2}-g_{1} g_{2} g_{3}=0 .
\end{aligned}
$$

The first-order derivative of $\Phi$ with respect to $g_{1}$ is found to be

$$
\begin{aligned}
\frac{\partial \Phi}{\partial g_{1}}= & \cos \frac{\phi_{2}}{2} \cos \frac{\phi_{3}}{2} \\
& -\left(g_{2} \cos \frac{\phi_{3}}{2}+g_{3} \cos \frac{\phi_{2}}{2}\right) \tan \frac{\phi_{1}}{2}-g_{2} g_{3} .
\end{aligned}
$$

The derivatives $\partial \Phi / \partial g_{2}$ and $\partial \Phi / \partial g_{3}$ can be obtained by cyclic permutations of eq. (61).

For a nonplanar structure, as in Figure 1, the new out-of-plane angle coordinate, $h$, depends on the related valence angles, $\phi_{1}, \phi_{2}$, and $\phi_{3}$. The area of a triangle defined by the three end points of $\mathbf{e}_{41}, \mathbf{e}_{42}$, and $\mathbf{e}_{43}$ is found to be (Heron's formula)

$$
\sqrt{s\left(s-2 g_{1}\right)\left(s-2 g_{2}\right)\left(s-2 g_{3}\right)}
$$

with $s \equiv g_{1}+g_{2}+g_{3}$ (Fig. 2). This should be the same as the total contribution from the three subtriangles due to projection of the three unit bond vectors on the triangle:

$$
t_{1} g_{1}+t_{2} g_{2}+t_{3} g_{3}
$$

where each $t_{j}(j=1,2,3)$ is defined by $t_{j}^{2} \equiv 1-$ $h^{2}-g_{j}^{2}$. Thus, the (nonlinear) redundancy relation between the new out-of-plane angle coordinate, $h$, and the related valence angles is found to be

$$
\begin{gathered}
\Phi=s\left(s-2 g_{1}\right)\left(s-2 g_{2}\right)\left(s-2 g_{3}\right) \\
-\left(t_{1} g_{1}+t_{2} g_{2}+t_{3} g_{3}\right)^{2}=0 .
\end{gathered}
$$

\section{Applications}

$\mathrm{NH}_{3}$

An isolated ammonia molecule has a pyramidal equilibrium structure whose geometry has $\mathbf{C}_{3 v}$ point symmetry. In terms of our new coordinates, its six genuine internal vibrations can be expressed 
by the following (symmetry) internal coordinates

$$
\begin{gathered}
\mathbf{R}^{1}\left(A_{1}\right)=\frac{1}{\sqrt{3}}\left(r_{41}+r_{42}+r_{43}\right) \\
\mathbf{R}^{2}\left(A_{1}\right)=h \\
\mathbf{R}^{3 \alpha}(E)=\frac{1}{\sqrt{6}}\left(2 r_{41}-r_{42}-r_{43}\right) \\
\mathbf{R}^{3 \beta}(E)=\frac{1}{\sqrt{2}}\left(r_{42}-r_{43}\right) \\
\mathbf{R}^{4 \alpha}(E)=\frac{1}{\sqrt{6}}\left(-g_{1}-g_{2}+2 g_{3}\right) \\
\mathbf{R}^{4 \beta}(E)=\frac{1}{\sqrt{2}}\left(g_{1}-g_{2}\right)
\end{gathered}
$$

where $r_{4 i}, g_{i}$, and $h$ represent the related bond lengths, bond angles, and the out-of-plane angle, respectively (see Fig. 1). Each doubly degenerate state is labeled by $\alpha$ and $\beta$.

An $a b$ initio calculation at the MP2/6-311++ $\mathrm{G}^{* *}$ level gives an optimized geometry of $r_{4 i}^{\mathrm{eq}}=$ $1.013 \AA, g_{i}^{\text {eq }}=0.8054\left(\phi_{i}^{\text {eq }}=107.29^{\circ}\right)$, and $h^{\text {eq }}=$ 0.3676 (out-of-plane angle of $\gamma^{\mathrm{eq}}=21.57^{\circ}$ with $h \equiv \sin \gamma, \omega^{\mathrm{eq}} \quad$ (Wilson-type $\left.)=59.90^{\circ}\right)$; frequencies of $\nu_{1}=3530\left(\mathrm{NH}\right.$ symmetric stretch, ss), $\nu_{2}=$ 1069 (out-of-plane deformation, umbrella mode), $\nu_{3}^{\alpha}=\nu_{3}^{\beta}=3682(\mathrm{NH}$ antisymmetric stretch, as), and $\nu_{4}^{\alpha}=\nu_{4}^{\beta}=1665 \mathrm{~cm}^{-1}$ (HNH bend). The outof-plane coordinate, $h$, has a significant interaction only with $\mathbf{R}^{1}$, and the related harmonic force constants are found to be $f_{11}=7.2210 \mathrm{mdyn} / \AA, f_{22}=$ 2.1735 mdyn $\AA, f_{12}=-0.9865$ mdyn. In a planar transition structure $\left(h^{\text {tr }}=0\right)$, which is optimized at $r_{4 i}^{\mathrm{tr}}=0.999 \AA$ and $g_{i}^{\mathrm{tr}}=0.8660\left(\phi_{i}^{\mathrm{tr}}=120.00^{\circ}\right)$ with the same basis set, the value of $f_{12}^{\text {tr }}$ is zero by symmetry and the diagonal force constant of the out-of-plane coordinate is found to be negative with $f_{22}^{\text {tr }}=-1.0468$ mdyn $\AA$, giving an imaginary vibrational frequency of $\nu_{2}^{\mathrm{tr}}=846 i \mathrm{~cm}^{-1}$. The calculated barrier height, which depends on the basis set choice, ${ }^{23,24}$ is found to be $1763 \mathrm{~cm}^{-1}(5.041$ $\mathrm{kcal} / \mathrm{mol}$ ).

The MP2/6-311 $++\mathrm{G}^{* *}$ potential energy surface was obtained by geometric optimizations at each $1^{\circ}$ increment of $\gamma$ from $0^{\circ}$ (planar orientation) to $60^{\circ}$. These $a b$ initio energy values are converted to ones in $h$, and the optimized potential energy parameters of eq. (23) are found to be $V_{2}=-27370$ and $V_{4}=106223 \mathrm{~cm}^{-1}$ with $t=$ 1.024 and $s=50$. The resulting model potential function (labeled by circles in Fig. 5) gives an excellent fit to this $a b$ initio energy surface in the range of $-\gamma^{\mathrm{eq}} \leq \gamma \leq \gamma^{\mathrm{eq}}$ (maximum deviation of $26 \mathrm{~cm}^{-1}$ ), although the deviation becomes significant outside of this region (it is higher than the $a b$ initio energy by 253, 1890, and $2644 \mathrm{~cm}^{-1}$ at $\gamma=$

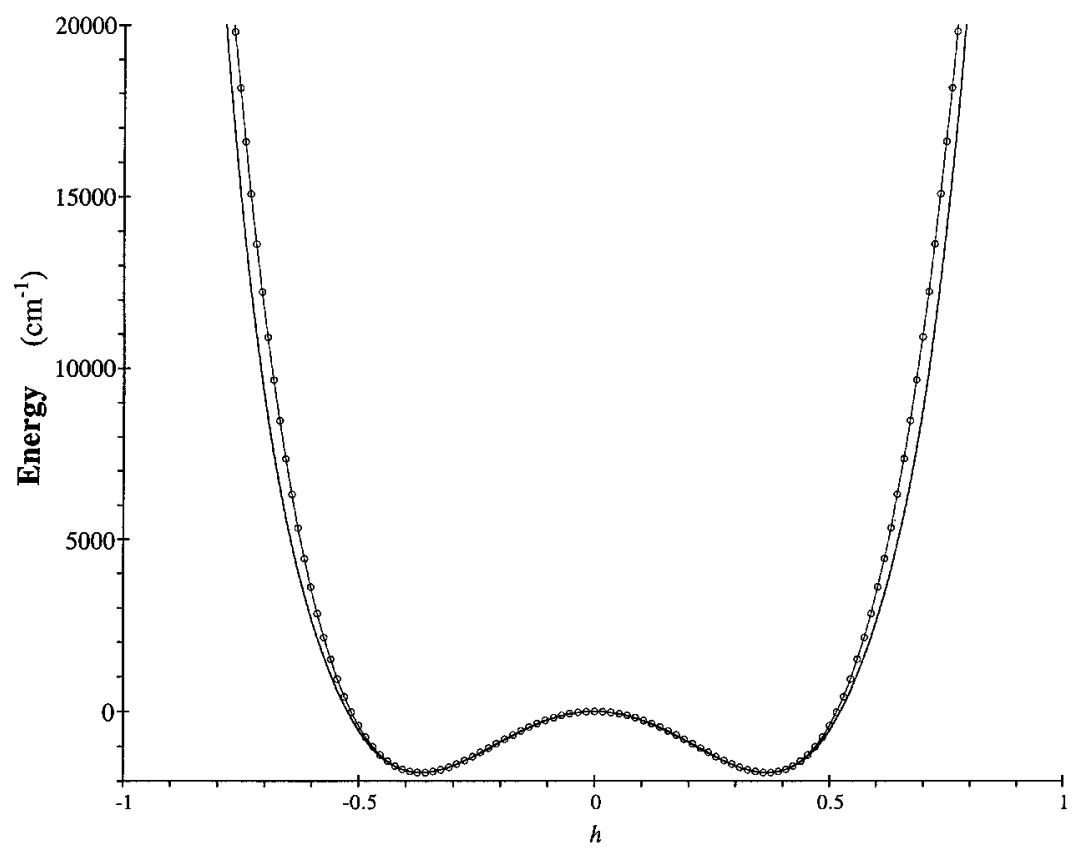

FIGURE 5. $A b$ initio MP2 $/ 6-311++\mathrm{G}^{* *}$ potential energy surface of an out-of-plane deformation for an isolated $\mathrm{NH}_{3}$ molecule, in $h$. The new model potential surface is labeled by circles. 
$32^{\circ}, 42^{\circ}$, and $52^{\circ}$, respectively). However, we believe our model potential function is good enough for typical MM and MD simulations.

One of the important features due to tunneling through the barrier in the symmetric double minima potential function is the vibrational splitting, $\Delta_{n}$, in the out-of-plane deformation mode. It was observed that $\Delta_{0} \approx 1, \Delta_{1} \approx 36, \Delta_{2} \approx 280-284, \Delta_{3}$ $\approx 511-512 \mathrm{~cm}^{-1}$ for an isolated ammonia molecule. ${ }^{25-27}$ In an extensive $a b$ initio study of this molecule by Rush and Wiberg, using a polynomial expansion for the potential, these splittings were found to be $\Delta_{0}=2, \Delta_{1}=77, \Delta_{2}=378, \Delta_{3}=$ $550 \mathrm{~cm}^{-1}$ at the MP2/6-311++ $\mathrm{G}^{* *}$ level. ${ }^{23}$ Our analytic form for the potential, Eq. (23), should provide a more complete description for this calculation.

\section{$\mathbf{H}_{2} \mathbf{O}$}

The symmetry elements of an isolated water molecule belong to the $\mathbf{C}_{2 v}$ point group. Its three genuine internal vibrations can be expressed by the following internal coordinates

$$
\begin{gathered}
\mathbf{R}^{1}\left(A_{1}\right)=\frac{1}{\sqrt{2}}\left(r_{21}+r_{23}\right) \\
\mathbf{R}^{2}\left(A_{1}\right)=g \\
\mathbf{R}^{3}\left(B_{1}\right)=\frac{1}{\sqrt{2}}\left(r_{21}-r_{23}\right)
\end{gathered}
$$

where $g$ represents the related bond-angle coordinate (see Fig. 3).

The $a b$ initio calculation at the MP2/6-311+ + $\mathrm{G}^{* *}$ level gives an equilibrium geometry of $r_{2 i}^{\mathrm{eq}}=$ $0.960 \AA$ and $g^{\text {eq }}=0.7851\left(\phi_{2}^{\text {eq }}=103.47^{\circ}\right)$; frequencies of $\nu_{1}=3885(\mathrm{OH} \mathrm{ss}), \nu_{2}=1629$ (HOH bend), $\nu_{3}=4003 \mathrm{~cm}^{-1}\left(\mathrm{OH}\right.$ as); force constants of $f_{11}=$ 8.5755, $f_{33}=8.8283$ mdyn $/ \AA, f_{22}=7.1676$ mdyn $\AA\left(0.6873 \mathrm{mdyn} \AA /(\mathrm{rad})^{2}\right.$ in $\left.\phi_{2}\right), f_{12}=1.1424$ mdyn. In the linear transition configuration $(g=$ $1)$, which has $\mathbf{D}_{\infty \circ h}$ symmetry and is optimized at $r_{2 i}^{\mathrm{tr}}=0.937 \AA$ with the same basis set, there is an additional bond-angle degree of freedom giving a doubly degenerate linear angle bend mode. Using the new linear angle coordinates with B-matrix elements being computed from eqs. (48)-(51), the corresponding diagonal force constants are found to be $f_{22}^{\alpha}=f_{22}^{\beta}=-0.6841 \mathrm{mdyn} \AA /(\mathrm{rad})^{2}$ with imaginary vibrational frequencies of $\nu_{2}^{\alpha}=\nu_{2}^{\beta}=$ $1720 i \mathrm{~cm}^{-1}$. The barrier height is calculated to be
$11276 \mathrm{~cm}^{-1}$ (cf. similar results ${ }^{24}$ ), which is too big for a splitting due to tunneling through the potential barrier to be observed in a usual infrared spectrum. However, some hot bands of an $\mathrm{H}_{2} \mathrm{O}$ molecule may be significantly affected by such a tunneling effect. Note that we can always construct a molecule-fixed coordinate system that is separated from external rotations, and a large-amplitude $\mathrm{HOH}$ inversion motion may be suitably described in such a molecule-fixed coordinate system.

The MP2/6-311 $++\mathrm{G}^{* *}$ potential energy surface (Fig. 6) was obtained by geometric optimizations at each $2.5^{\circ}$ increment of $\phi_{2}$ from $40^{\circ}$ to $180^{\circ}$ $(g=1)$. From these $a b$ initio results, the optimized potential energy parameters of eq. (40) are found to be $V_{1}=-84559$ and $V_{2}=158517 \mathrm{~cm}^{-1}$ with $t=0.859$ and $s=12$. Overall, the resulting model potential function (labeled by circles in Fig. 6) gives an excellent fit to the $a b$ initio energy surface [maximum deviation of $278 \mathrm{~cm}^{-1}$ at $\phi_{2}=157.5^{\circ}$ in the range of $\left.-\left(\phi_{2}^{\mathrm{eq}}+30^{\circ}\right) \leq \phi_{2} \leq\left(\phi_{2}^{\mathrm{eq}}+30^{\circ}\right)\right]$. Although the potential surface in terms of $\phi_{2}$ has a similar shape to that of an out-of-plane coordinate, it has a discontinuous first derivative at a linear configuration in terms of the new angle bend coordinate, $g$ (see Fig. 6b).

For an $\mathrm{Si}-\mathrm{O}-\mathrm{Si}$ angle bend potential function of an isolated disiloxane $\left(\mathrm{H}_{3} \mathrm{SiOSiH}_{3}\right)$ molecule, Koput $^{28}$ introduced a Lorentzian hump of

$$
V_{\rho}=\frac{B_{h} f_{\phi \phi}\left(\rho^{2}-\rho_{\mathrm{eq}}^{2}\right)^{2}}{f_{\phi \phi} \rho_{\mathrm{eq}}^{4}+\left(8 B_{h}-f_{\phi \phi} \rho_{\mathrm{eq}}^{2}\right) \rho^{2}}
$$

with $\rho \equiv \pi-\phi_{2}$. As a comparison with our potential function, the potential surface based on eq. (74) is also plotted in Figure 6a (labeled by triangles). Although such a Lorentzian hump may well reproduce the $a b$ initio potential energy surface of an $\mathrm{Si}-\mathrm{O}-\mathrm{Si}$ angle bend potential for a disiloxane molecule, which has a very small barrier, ${ }^{29,30}$ it is far from reproducing the angle bend potential energy surface of an $\mathrm{H}_{2} \mathrm{O}$ molecule. In view of the expected very small barrier in an $\mathrm{Si}-\mathrm{O}-\mathrm{Si}$ angle bend potential, a tunneling effect should be taken into consideration for a more accurate spectroscopic analysis of the disiloxane molecule.

\section{$\mathrm{CO}_{2}$}

The symmetry elements of an isolated carbon dioxide molecule belong to the $\mathbf{D}_{\infty h}$ group. Its four genuine internal vibrations can be expressed by 


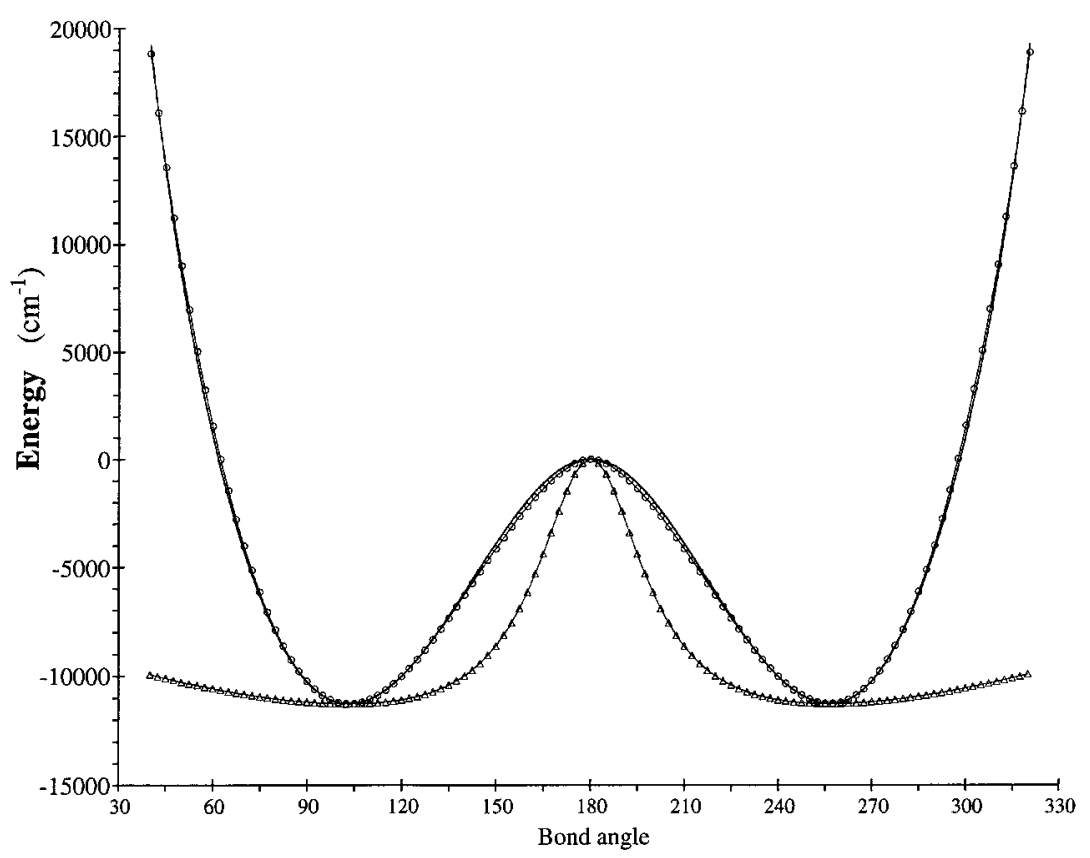

(a)

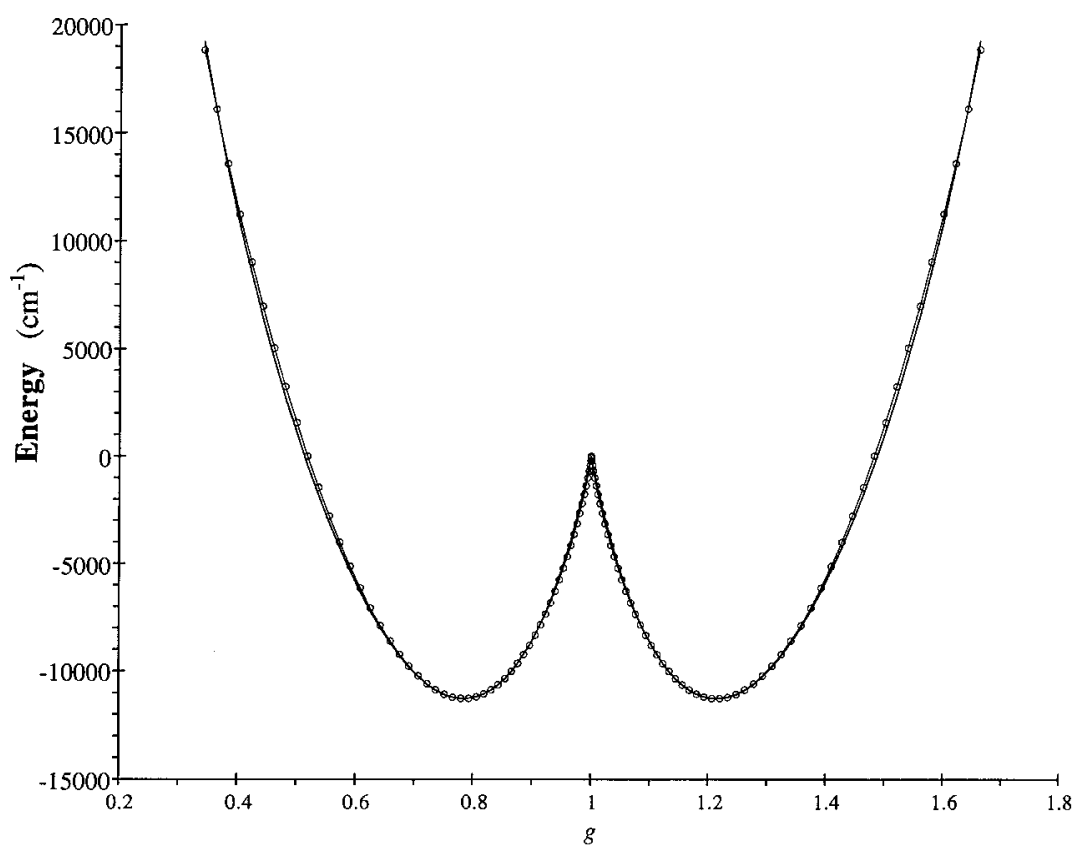

(b)

FIGURE 6. Ab initio MP2 $/ 6-311++\mathrm{G}^{* *}$ potential energy surface of an $\mathrm{HOH}$ bend of an isolated $\mathrm{H}_{2} \mathrm{O}$ molecule. The new model potential surface is labeled by circles; (a) in bond angle (the surface based on Koput's potential energy function is labeled by triangles); (b) in $g$. 
the following internal coordinates

$$
\begin{gathered}
\mathbf{R}^{1}\left(\Sigma_{g}^{+}\right)=\frac{1}{\sqrt{2}}\left(r_{21}+r_{23}\right) \\
\mathbf{R}^{2 \alpha}\left(\Pi_{u}\right)=\theta_{u} \\
\mathbf{R}^{2 \beta}\left(\Pi_{u}\right)=\theta_{w} \\
\mathbf{R}^{3}\left(\Sigma_{u}^{+}\right)=\frac{1}{\sqrt{2}}\left(r_{21}-r_{23}\right)
\end{gathered}
$$

where $\theta_{u}$ and $\theta_{w}$ represent the related linear angle coordinates whose B-matrix elements can be obtained from eqs. (48)-(51) (see Fig. 4). The ab initio calculation at the MP2/6-311+ $+\mathrm{G}^{* *}$ level gives a linear equilibrium geometry of $r_{2 i}^{\mathrm{eq}}=1.170 \AA$; frequencies of $\nu_{1}=1335(\mathrm{CO} \mathrm{ss}), \nu_{2}^{\alpha}=\nu_{2}^{\beta}=655$ (OCO bend), $\nu_{3}=2432 \mathrm{~cm}^{-1}$ (CO as); force constants of $f_{11}=16.8071, f_{33}=15.2146 \mathrm{mdyn} / \AA$, and $f_{22}^{\alpha}=f_{22}^{\beta}=0.7553$ mdyn $\AA /(\operatorname{rad})^{2}$.

The MP2/6-311 + $+\mathrm{G}^{* *}$ potential energy surface was obtained by geometric optimizations at each $2.5^{\circ}$ increment of $\phi_{2}$ (see Fig. 3) from $90^{\circ}$ to $180^{\circ}\left(g^{\text {eq }}=1\right)$. Although the potential surface is close to a quadratic form in terms of $\phi_{2}$, it is close to a linear form in terms of the new angle bend coordinate, $g$ (Fig. 7). The potential energy parameters of eq. (40) optimized to the calculated $a b$ initio energy surface are found to be $V_{1}=152091$ and $V_{2}=215000 \mathrm{~cm}^{-1}$ with $t=1$ and $s=10$. The resulting model potential function in terms of $g$ (labeled by circles in Fig. 7) gives an excellent fit to the $a b$ initio energy surface (maximum deviation of $2 \mathrm{~cm}^{-1}$ in the range of $150^{\circ} \leq \phi_{2} \leq 210^{\circ}$ ), showing that the new angle bend coordinate defined by eq. (34) is also a good coordinate for MM and MD simulations of a molecule with a linear equilibrium structure.

\section{Conclusions}

The Wilson-type conventional out-of-plane coordinate has singularities in its Cartesian derivatives at a value of $\pm \pi / 2$, causing the force acting on a related atom to be ill-defined. The conventional improper torsion coordinate, which is commonly used in MM and MD simulations to represent an out-of-plane deformation, is found to be dependent on the related internal bond length for nonplanar configurations. We have introduced a new out-of-plane internal coordinate, which is well defined in all physically admissible molecular configurations. Because it is already in a symmetric form, it does not require an additional symmetrization for MM or MD simulations. A newly introduced potential energy function in terms of this coordinate is found to excellently reproduce

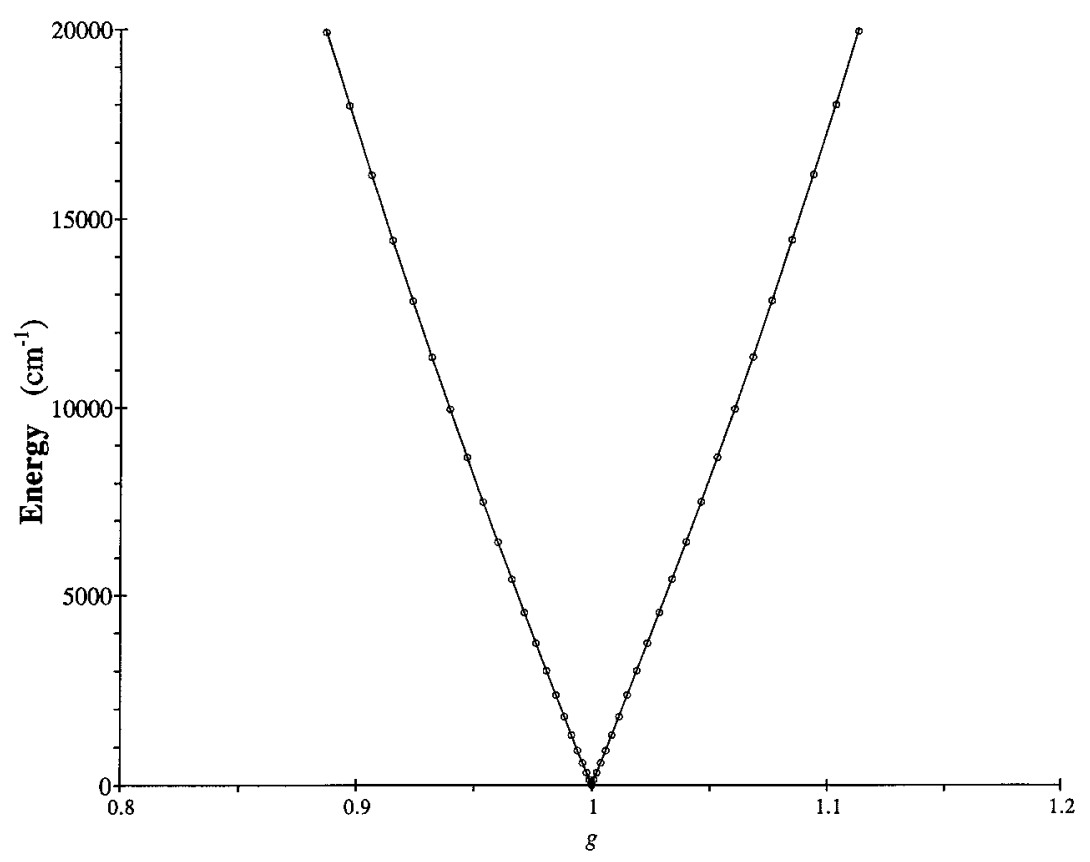

FIGURE 7. $A b$ initio MP2 $/ 6-311++\mathrm{G}^{* *}$ potential energy surface of an $\mathrm{OCO}$ bend of an isolated $\mathrm{CO}_{2}$ molecule, in $g$. The new model potential surface is labeled by circles. 
the ab initio MP2/6-311+ $+\mathrm{G}^{* *}$ potential energy surface of an isolated $\mathrm{NH}_{3}$ molecule.

A new nonlinear bond-angle coordinate is introduced to avoid the problem resulting from the singularity in Cartesian derivatives of the conventional bond-angle coordinate ${ }^{8}$ for a linear molecular configuration. The related new potential energy function, which is essentially a parallel shift of the new out-of-plane potential function, is found to give an excellent fit to the $a b$ initio MP2/6-311 $++G^{* *}$ potential energy surface of an isolated $\mathrm{H}_{2} \mathrm{O}$ molecule. For a vibrational analysis of a linear equilibrium structure, a new linear angle coordinate is introduced based on a torsion angle definition, which is faster and simpler in computation than the conventional linear angle definition..$^{20}$ It is shown that the new bond-angle coordinate is also a suitable coordinate for MM or MD simulations of a linear equilibrium structure.

The general potential energy surface of an angle deformation of a nonlinear equilibrium structure is found to have symmetric double minima, ${ }^{18}$ as in the case of the $\mathrm{NH}_{3}$ molecule. For a molecule with a small-angle bend potential barrier, the effect of tunneling through the potential barrier may be observable in infrared or Raman spectra as a splitting in the related angle bend mode.

\section{Acknowledgment}

This research was supported by NSF Grants MCB-9601006 and DMR-9627786. Additional support was provided by the Air Force Research Laboratory/Materials \& Manufacturing Directorate, and by the Common High-Performance Software Support Initiative of the Department of Defense High-Performance Computing Program.

\section{Appendix}

\section{BASIC DERIVATIVES WITH RESPECT TO DISTANCE VECTORS}

As we have already shown, the B-matrix elements of an internal coordinate can easily be obtained from some derivatives with respect to distance vectors through eqs. (4)-(5). The required derivatives are actually categorized into two types, which can be programmed into two subroutines. The first one is related to each distance vector itself, and the necessary quantities are derivatives of each unit distance vector with respect to its distance vector:

$$
\begin{gathered}
\frac{\partial \mathbf{e}_{12}}{\partial \mathbf{x}_{12}^{k}}=\frac{1}{r_{12}}\left(\hat{\mathbf{k}}-\mathbf{e}_{12}^{k} \mathbf{e}_{12}\right) \\
\frac{\partial^{2} \mathbf{e}_{12}}{\partial \mathbf{x}_{12}^{j} \partial \mathbf{x}_{12}^{k}}=\frac{-1}{r_{12}}\left(\mathbf{e}_{12}^{j} \frac{\partial \mathbf{e}_{12}}{\partial \mathbf{x}_{12}^{k}}+\mathbf{e}_{12}^{k} \frac{\partial \mathbf{e}_{12}}{\partial \mathbf{x}_{12}^{j}}+\frac{\partial \mathbf{e}_{12}^{k}}{\partial \mathbf{x}_{12}^{j}} \mathbf{e}_{12}\right) \\
\frac{\partial^{3} \mathbf{e}_{12}}{\partial \mathbf{x}_{12}^{i} \partial \mathbf{x}_{12}^{j} \partial \mathbf{x}_{12}^{k}} \\
=\frac{-1}{r_{12}}\left(\mathbf{e}_{12}^{i} \frac{\partial^{2} \mathbf{e}_{12}}{\partial \mathbf{x}_{12}^{j} \partial \mathbf{x}_{12}^{k}}+\mathbf{e}_{12}^{j} \frac{\partial^{2} \mathbf{e}_{12}}{\partial \mathbf{x}_{12}^{k} \partial \mathbf{x}_{12}^{i}}\right. \\
+\mathbf{e}_{12}^{k} \frac{\partial^{2} \mathbf{e}_{12}}{\partial \mathbf{x}_{12}^{i}}+\frac{\partial \mathbf{e}_{12}^{j}}{\partial \mathbf{x}_{12}^{i}} \frac{\partial \mathbf{e}_{12}}{\partial \mathbf{x}_{12}^{k}}+\frac{\partial \mathbf{e}_{12}^{k}}{\partial \mathbf{x}_{12}^{j}} \frac{\partial \mathbf{e}_{12}}{\partial \mathbf{x}_{12}^{i}} \\
\left.+\frac{\partial \mathbf{e}_{12}^{i}}{\partial \mathbf{x}_{12}^{k}} \frac{\partial \mathbf{e}_{12}}{\partial \mathbf{x}_{12}^{j}}+\frac{\partial^{2} \mathbf{e}_{12}^{k}}{\partial \mathbf{x}_{12}^{i} \partial \mathbf{x}_{12}^{j}} \mathbf{e}_{12}\right)
\end{gathered}
$$

where a Cartesian unit vector $\hat{\mathbf{k}}$ is defined as $\hat{\mathbf{1}} \equiv(1,0,0), \hat{\mathbf{2}} \equiv(0,1,0)$, and $\hat{\mathbf{3}} \equiv(0,0,1)$.

The second one is related to a bond angle internal coordinate (Fig. 3), where the useful quantities are derivatives of a unit vector $\mathbf{u}$ defined by

$$
\mathbf{u} \equiv \frac{\mathbf{e}_{21} \times \mathbf{e}_{23}}{\sin \phi}
$$

where $\phi\left(\cos \phi=\mathbf{e}_{12} \cdot \mathbf{e}_{23}\right)$ is an angle between $\mathbf{x}_{21}$ and $\mathbf{x}_{23}$. Using the following relations

$$
r_{21} \frac{\partial \cos \phi}{\partial \mathbf{x}_{21}^{k}}=\mathbf{e}_{23}^{k}-\mathbf{e}_{21}^{k} \cos \phi=-\sin \phi\left(\mathbf{e}_{21} \times \mathbf{u}\right)^{k}
$$

$$
r_{23} \frac{\partial \cos \phi}{\partial \mathbf{x}_{23}^{k}}=\mathbf{e}_{21}^{k}-\mathbf{e}_{23}^{k} \cos \phi=-\sin \phi\left(\mathbf{u} \times \mathbf{e}_{23}\right)^{k}
$$

$$
\begin{aligned}
r_{21} \frac{\partial \sin \phi}{\partial \mathbf{x}_{21}^{k}} & =-\left(\mathbf{u} \times \mathbf{e}_{23}\right)^{k}-\sin \phi \mathbf{e}_{21}^{k} \\
& =\cos \phi\left(\mathbf{e}_{21} \times \mathbf{u}\right)^{k} \\
r_{23} \frac{\partial \sin \phi}{\partial \mathbf{x}_{23}^{k}} & =-\left(\mathbf{e}_{21} \times \mathbf{u}\right)^{k}-\sin \phi \mathbf{e}_{23}^{k} \\
& =\cos \phi\left(\mathbf{u} \times \mathbf{e}_{23}\right)^{k},
\end{aligned}
$$

the desired derivates of $\mathbf{u}$ with respect to the related distance vectors are found to be

$$
\frac{\partial \mathbf{u}}{\partial \mathbf{x}_{21}^{k}}=\frac{1}{r_{21} \sin \phi}\left\{\hat{\mathbf{k}} \times \mathbf{e}_{23}+\left(\mathbf{u} \times \mathbf{e}_{23}\right)^{k} \mathbf{u}\right\}
$$


$\frac{\partial \mathbf{u}}{\partial \mathbf{x}_{23}^{k}}=\frac{1}{r_{23} \sin \phi}\left\{\mathbf{e}_{21} \times \hat{\mathbf{k}}+\left(\mathbf{e}_{21} \times \mathbf{u}\right)^{k} \mathbf{u}\right\} \quad(\mathrm{a} 10)$

$$
\begin{aligned}
\frac{\partial^{2} \mathbf{u}}{\partial \mathbf{x}_{21}^{j} \partial \mathbf{x}_{21}^{k}}= & \frac{1}{r_{21} \sin \phi}\left\{\left(\mathbf{u} \times \mathbf{e}_{23}\right)^{j} \frac{\partial \mathbf{u}}{\partial \mathbf{x}_{21}^{k}}\right. \\
& +\left(\mathbf{u} \times \mathbf{e}_{23}\right)^{k} \frac{\partial \mathbf{u}}{\partial \mathbf{x}_{21}^{j}} \\
& \left.+\left(\frac{\partial \mathbf{u}}{\partial \mathbf{x}_{21}^{j}} \times \mathbf{e}_{23}\right)^{k} \mathbf{u}\right\} \\
\frac{\partial^{2} \mathbf{u}}{\partial \mathbf{x}_{23}^{j} \partial \mathbf{x}_{23}^{k}}= & \frac{1}{r_{23} \sin \phi}\left\{\left(\mathbf{e}_{21} \times \mathbf{u}\right)^{j} \frac{\partial \mathbf{u}}{\partial \mathbf{x}_{23}^{k}}\right. \\
& +\left(\mathbf{e}_{21} \times \mathbf{u}\right)^{k} \frac{\partial \mathbf{u}}{\partial \mathbf{x}_{23}^{j}} \\
& \left.+\left(\mathbf{e}_{21} \times \frac{\partial \mathbf{u}}{\partial \mathbf{x}_{23}^{j}}\right)^{k} \mathbf{u}\right\}
\end{aligned}
$$

$$
\begin{aligned}
\frac{\partial^{2} \mathbf{u}}{\partial \mathbf{x}_{23}^{j} \partial \mathbf{x}_{21}^{k}}= & \frac{1}{r_{23} r_{21} \sin \phi}\left\{\left(\mathbf{e}_{21} \times \mathbf{u}\right)^{j} r_{21} \frac{\partial \mathbf{u}}{\partial \mathbf{x}_{21}^{k}}\right. \\
& +\left(\mathbf{u} \times \mathbf{e}_{23}\right)^{k} r_{23} \frac{\partial \mathbf{u}}{\partial \mathbf{x}_{23}^{j}}+\hat{\mathbf{k}} \times \hat{\mathbf{j}} \\
& \left.+\left(r_{23} \frac{\partial \mathbf{u}}{\partial \mathbf{x}_{23}^{j}} \times \mathbf{e}_{23}+\mathbf{u} \times \hat{\mathbf{j}}\right)^{k} \mathbf{u}\right\}
\end{aligned}
$$

$$
\begin{aligned}
\frac{\partial^{3} \mathbf{u}}{\partial \mathbf{x}_{21}^{i} \partial \mathbf{x}_{21}^{j} \partial \mathbf{x}_{21}^{k}} & \frac{1}{r_{21} \sin \phi}\left\{\left(\mathbf{u} \times \mathbf{e}_{23}\right)^{i} \frac{\partial^{2} \mathbf{u}}{\partial \mathbf{x}_{21}^{j} \partial \mathbf{x}_{21}^{k}}\right. \\
& +\left(\mathbf{u} \times \mathbf{e}_{23}\right)^{j} \frac{\partial^{2} \mathbf{u}}{\partial \mathbf{x}_{21}^{k} \partial \mathbf{x}_{21}^{i}} \\
& +\left(\mathbf{u} \times \mathbf{e}_{23}\right)^{k} \frac{\partial^{2} \mathbf{u}}{\partial \mathbf{x}_{21}^{i} \partial \mathbf{x}_{21}^{j}} \\
& +\left(\frac{\partial \mathbf{u}}{\partial \mathbf{x}_{21}^{i}} \times \mathbf{e}_{23}\right)^{j} \frac{\partial \mathbf{u}}{\partial \mathbf{x}_{21}^{k}} \\
& +\left(\frac{\partial \mathbf{u}}{\partial \mathbf{x}_{21}^{j}} \times \mathbf{e}_{23}\right)^{k} \frac{\partial \mathbf{u}}{\partial \mathbf{x}_{21}^{i}} \\
& +\left(\frac{\partial \mathbf{u}}{\partial \mathbf{x}_{21}^{k}} \times \mathbf{e}_{23}\right)^{i} \frac{\partial \mathbf{u}}{\partial \mathbf{x}_{21}^{j}} \\
& \left.+\left(\frac{\partial^{2} \mathbf{u}}{\partial \mathbf{x}_{21}^{i} \partial \mathbf{x}_{21}^{j}} \times \mathbf{e}_{23}\right)^{k} \mathbf{u}\right\}
\end{aligned}
$$

$$
\begin{aligned}
\frac{\partial^{3} \mathbf{u}}{\partial \mathbf{x}_{23}^{i} \partial \mathbf{x}_{23}^{j} \partial \mathbf{x}_{23}^{k}} & \frac{1}{r_{23} \sin \phi}\left\{\left(\mathbf{e}_{21} \times \mathbf{u}\right)^{i} \frac{\partial^{2} \mathbf{u}}{\partial \mathbf{x}_{23}^{j} \partial \mathbf{x}_{23}^{k}}\right. \\
& +\left(\mathbf{e}_{21} \times \mathbf{u}\right)^{j} \frac{\partial^{2} \mathbf{u}}{\partial \mathbf{x}_{23}^{k} \partial \mathbf{x}_{23}^{i}} \\
& +\left(\mathbf{e}_{12} \times \mathbf{u}\right)^{k} \frac{\partial^{2} \mathbf{u}}{\partial \mathbf{x}_{23}^{i} \partial \mathbf{x}_{23}^{j}} \\
& +\left(\mathbf{e}_{21} \times \frac{\partial \mathbf{u}}{\partial \mathbf{x}_{23}^{i}}\right)^{j} \frac{\partial \mathbf{u}}{\partial \mathbf{x}_{23}^{k}} \\
& +\left(\mathbf{e}_{21} \times \frac{\partial \mathbf{u}}{\partial \mathbf{x}_{23}^{j}}\right)^{k} \frac{\partial \mathbf{u}}{\partial \mathbf{x}_{23}^{i}} \\
& +\left(\mathbf{e}_{21} \times \frac{\partial \mathbf{u}}{\partial \mathbf{x}_{23}^{k}}\right)^{i} \frac{\partial \mathbf{u}}{\partial \mathbf{x}_{23}^{j}} \\
& \left.+\left(\mathbf{e}_{21} \times \frac{\partial^{2} \mathbf{u}}{\partial \mathbf{x}_{23}^{i} \partial \mathbf{x}_{23}^{j}}\right)^{k} \mathbf{u}\right\}
\end{aligned}
$$

$\frac{\partial^{3} \mathbf{u}}{\partial \mathbf{x}_{23}^{i} \partial \mathbf{x}_{21}^{j} \partial \mathbf{x}_{21}^{k}}$

$$
=\frac{1}{r_{23} r_{21} \sin \phi}
$$

$$
\times\left\{\left(\mathbf{e}_{21} \times \mathbf{u}\right)^{i} r_{21} \frac{\partial^{2} \mathbf{u}}{\partial \mathbf{x}_{21}^{j} \partial \mathbf{x}_{21}^{k}}\right.
$$$$
+\left(\mathbf{u} \times \mathbf{e}_{23}\right)^{j} r_{23} \frac{\partial^{2} \mathbf{u}}{\partial \mathbf{x}_{23}^{i} \partial \mathbf{x}_{21}^{k}}
$$$$
+\left(\mathbf{u} \times \mathbf{e}_{23}\right)^{k} r_{23} \frac{\partial^{2} \mathbf{u}}{\partial \mathbf{x}_{23}^{i} \partial \mathbf{x}_{21}^{j}}
$$$$
+\left(r_{23} \frac{\partial \mathbf{u}}{\partial \mathbf{x}_{23}^{i}} \times \mathbf{e}_{23}+\mathbf{u} \times \hat{\mathbf{i}}\right)^{j} \frac{\partial \mathbf{u}}{\partial \mathbf{x}_{21}^{k}}
$$$$
+\left(r_{23} \frac{\partial \mathbf{u}}{\partial \mathbf{x}_{23}^{i}} \times \mathbf{e}_{23}+\mathbf{u} \times \hat{\mathbf{i}}\right)^{k} \frac{\partial \mathbf{u}}{\partial \mathbf{x}_{21}^{j}}
$$$$
+\left(\frac{\partial \mathbf{u}}{\partial \mathbf{x}_{21}^{j}} \times \mathbf{e}_{23}\right)^{k} r_{23} \frac{\partial \mathbf{u}}{\partial \mathbf{x}_{23}^{i}}
$$$$
\left.+\left(r_{23} \frac{\partial^{2} \mathbf{u}}{\partial \mathbf{x}_{23}^{i} \partial \mathbf{x}_{21}^{j}} \times \mathbf{e}_{23}+\frac{\partial \mathbf{u}}{\partial \mathbf{x}_{21}^{j}} \times \hat{\mathbf{i}}\right)^{k} \mathbf{u}\right\}
$$ 


$$
\begin{aligned}
\frac{\partial^{3} \mathbf{u}}{\partial \mathbf{x}_{23}^{i} \partial \mathbf{x}_{23}^{j} \partial \mathbf{x}_{21}^{k}} & \frac{1}{r_{23} r_{21} \sin \phi} \\
& \times\left\{\left(\mathbf{e}_{21} \times \mathbf{u}\right)^{i} r_{21} \frac{\partial^{2} \mathbf{u}}{\partial \mathbf{x}_{23}^{j} \partial \mathbf{x}_{21}^{k}}\right. \\
& +\left(\mathbf{e}_{21} \times \mathbf{u}\right)^{j} r_{21} \frac{\partial^{2} \mathbf{u}}{\partial \mathbf{x}_{23}^{i} \partial \mathbf{x}_{21}^{k}} \\
& +\left(\mathbf{u} \times \mathbf{e}_{23}\right)^{k} r_{23} \frac{\partial^{2} \mathbf{u}}{\partial \mathbf{x}_{23}^{i} \partial \mathbf{x}_{23}^{j}} \\
& +\left(\mathbf{e}_{21} \times r_{21} \frac{\partial \mathbf{u}}{\partial \mathbf{x}_{21}^{k}}+\hat{\mathbf{k}} \times \mathbf{u}\right)^{i} \frac{\partial \mathbf{u}}{\partial \mathbf{x}_{23}^{j}} \\
& +\left(\mathbf{e}_{21} \times r_{21} \frac{\partial \mathbf{u}}{\partial \mathbf{x}_{21}^{k}}+\hat{\mathbf{k}} \times \mathbf{u}\right)^{j} \frac{\partial \mathbf{u}}{\partial \mathbf{x}_{23}^{i}} \\
& +\left(\mathbf{e}_{21} \times \frac{\partial \mathbf{u}}{\partial \mathbf{x}_{23}^{i}}\right)^{j} r_{21} \frac{\partial \mathbf{u}}{\partial \mathbf{x}_{21}^{k}} \\
& \left.+\left(\mathbf{e}_{21} \times r_{21} \frac{\partial^{2} \mathbf{u}}{\partial \mathbf{x}_{23}^{i} \partial \mathbf{x}_{21}^{k}}+\hat{\mathbf{k}} \times \frac{\partial \mathbf{u}}{\partial \mathbf{x}_{23}^{i}}\right)^{j} \mathbf{u}\right\}
\end{aligned}
$$

\section{TORSION COORDINATE}

The expression for the B-matrix elements of a torsion angle can be formulated in a compact way if we separate the original structure into two independent bond-angle structures, with $\mathbf{x}_{23}$ and $\mathbf{x}_{32}$ being treated as independent of each other (the total related number of bonds being four instead of three) (Fig. 8); the required $\mathbf{K}_{\nu}^{b}$ elements are given by

$$
\mathbf{K}=\left(\begin{array}{rrrr}
1 & -1 & 0 & 0 \\
0 & -1 & 1 & 0 \\
0 & 1 & -1 & 0 \\
0 & 0 & -1 & 1
\end{array}\right)
$$

If we define $\mathbf{u}_{2} \equiv \mathbf{e}_{23} \times \mathbf{e}_{21} / \sin \phi_{2}$ and $\mathbf{u}_{3} \equiv \mathbf{e}_{34}$ $\times \mathbf{e}_{32} / \sin \phi_{3}$ (unit vectors), then a torsion angle, $\tau$, can be defined by

$$
\cos \tau=\mathbf{u}_{2} \cdot \mathbf{u}_{3}
$$

The desired derivatives with respect to the related
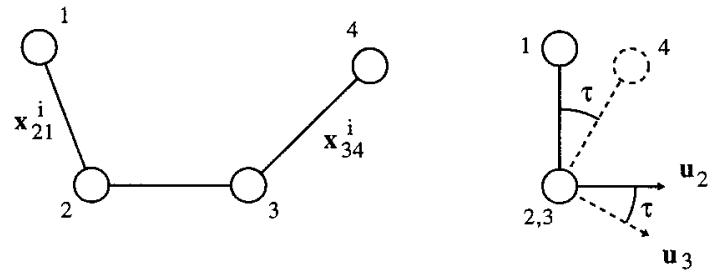

$$
\mathbf{u}_{2} \equiv\left(\mathbf{e}_{23} \times \mathbf{e}_{21}\right) / \sin \phi_{2}
$$

$\mathbf{u}_{3} \equiv\left(\mathbf{e}_{34} \times \mathbf{e}_{32}\right) / \sin \phi_{3}$

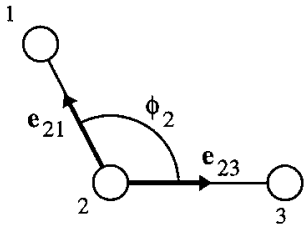

$\oplus$

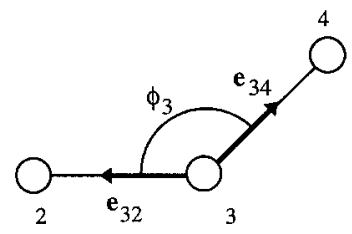

FIGURE 8. Torsion angle coordinate.

distance vectors are found to be

$$
\begin{gathered}
\frac{\partial \tau}{\partial \mathbf{x}_{21}^{k}}=\frac{-1}{R_{a}} \mathbf{u}_{2}^{k}, \quad R_{a} \equiv r_{21} G_{2}, \quad G_{2} \equiv \sin \phi_{2} \quad(\mathrm{a} 2 \\
\frac{\partial \tau}{\partial \mathbf{x}_{23}^{k}}=\frac{1}{R_{b}} F_{2} \mathbf{u}_{2}^{k}, \quad F_{2} \equiv \cos \phi_{2}, \quad R_{b} \equiv r_{23} G_{2}
\end{gathered}
$$

$$
\frac{\partial \tau}{\partial \mathbf{x}_{32}^{k}}=\frac{-1}{R_{c}} F_{3} \mathbf{u}_{3}^{k}, \quad F_{3} \equiv \cos \phi_{3}, \quad R_{c} \equiv r_{32} G_{3},
$$

$$
G_{3} \equiv \sin \phi_{3}
$$

$$
\frac{\partial \tau}{\partial \mathbf{x}_{34}^{k}}=\frac{1}{R_{d}} \mathbf{u}_{3}^{k}, \quad R_{d} \equiv r_{34} G_{3}
$$

$$
\begin{gathered}
\frac{\partial^{2} \tau}{\partial \mathbf{x}_{21}^{j} \partial \mathbf{x}_{21}^{k}}=\frac{-1}{R_{a}^{2}}\left\{\mathbf{v}_{2}^{j} \mathbf{u}_{2}^{k}+\left(\mathbf{e}_{23} \times \hat{\mathbf{j}}\right)^{k}\right\}, \\
\mathbf{v}_{1} \equiv 2\left(\mathbf{u}_{2} \times \mathbf{e}_{21}\right), \quad \mathbf{v}_{2} \equiv 2\left(\mathbf{e}_{23} \times \mathbf{u}_{2}\right)
\end{gathered}
$$

$$
\begin{aligned}
& \frac{\partial^{2} \tau}{\partial \mathbf{x}_{34}^{j} \partial \mathbf{x}_{34}^{k}}=\frac{1}{R_{d}^{2}}\left\{\mathbf{v}_{3}^{j} \mathbf{u}_{3}^{k}+\left(\hat{\mathbf{j}} \times \mathbf{e}_{32}\right)^{k}\right\}, \\
& \mathbf{v}_{3} \equiv 2\left(\mathbf{u}_{3} \times \mathbf{e}_{32}\right), \quad \mathbf{v}_{4} \equiv 2\left(\mathbf{e}_{34} \times \mathbf{u}_{3}\right)
\end{aligned}
$$

$$
\begin{gathered}
\frac{\partial^{2} \tau}{\partial \mathbf{x}_{23}^{j} \partial \mathbf{x}_{21}^{k}}=\frac{-1}{R_{b} R_{a}}\left\{\mathbf{w}_{21}^{j} \mathbf{u}_{2}^{k}+\left(\hat{\mathbf{j}} \times \mathbf{e}_{21}\right)^{k}\right\}, \\
\mathbf{w}_{21} \equiv \mathbf{v}_{1}+G_{2} \mathbf{e}_{23}
\end{gathered}
$$

$$
\begin{gathered}
\frac{\partial^{2} \tau}{\partial \mathbf{x}_{32}^{j} \partial \mathbf{x}_{34}^{k}}=\frac{1}{R_{c} R_{d}}\left\{\mathbf{w}_{34}^{j} \mathbf{u}_{3}^{k}+\left(\mathbf{e}_{34} \times \hat{\mathbf{j}}\right)^{k}\right\}, \\
\mathbf{w}_{34} \equiv \mathbf{v}_{4}+G_{3} \mathbf{e}_{32}
\end{gathered}
$$




$$
\begin{aligned}
& \frac{\partial^{2} \tau}{\partial \mathbf{x}_{23}^{j} \partial \mathbf{x}_{23}^{k}}=\frac{1}{R_{b}^{2}}\left\{\mathbf{w}_{22}^{j} \mathbf{u}_{2}^{k}+F_{2}\left(\hat{\mathbf{j}} \times \mathbf{e}_{21}\right)^{k}\right\}, \\
& \mathbf{w}_{22} \equiv F_{2} \mathbf{v}_{1}+G_{2} r_{23} \frac{\partial F_{2}}{\partial \mathbf{x}_{23}} \\
& \frac{\partial^{2} \tau}{\partial \mathbf{x}_{32}^{j} \partial \mathbf{x}_{32}^{k}}=\frac{-1}{R_{c}^{2}}\left\{\mathbf{w}_{33}^{j} \mathbf{u}_{3}^{k}+F_{3}\left(\mathbf{e}_{34} \times \hat{\mathbf{j}}\right)^{k}\right\}, \\
& \mathbf{w}_{33} \equiv F_{3} \mathbf{v}_{4}+G_{3} r_{32} \frac{\partial F_{3}}{\partial \mathbf{x}_{32}} \\
& \frac{\partial^{3} \boldsymbol{\tau}}{\partial \mathbf{x}_{21}^{i} \partial \mathbf{x}_{21}^{j} \partial \mathbf{x}_{21}^{k}}=\frac{-1}{R_{a}^{3}}\left\{2\left(\mathbf{v}_{2}^{i} \mathbf{v}_{2}^{j}+\mathbf{e}_{23}^{i} \mathbf{e}_{23}^{j}-\delta^{i j}\right) \mathbf{u}_{2}^{k}\right. \\
& \left.+\mathbf{v}_{2}^{i}\left(\mathbf{e}_{23} \times \hat{\mathbf{j}}\right)^{k}+\mathbf{v}_{2}^{i}\left(\mathbf{e}_{23} \times \hat{\mathbf{i}}\right)^{k}\right\} \\
& \frac{\partial^{3} \tau}{\partial \mathbf{x}_{34}^{i} \partial \mathbf{x}_{34}^{j} \partial \mathbf{x}_{34}^{k}}=\frac{1}{R_{d}^{3}}\left\{2\left(\mathbf{v}_{3}^{i} \mathbf{v}_{3}^{j}+\mathbf{e}_{32}^{i} \mathbf{e}_{32}^{j}-\delta^{i j}\right) \mathbf{u}_{3}^{k}\right. \\
& \left.+\mathbf{v}_{3}^{i}\left(\hat{\mathbf{j}} \times \mathbf{e}_{32}\right)^{k}+\mathbf{v}_{3}^{j}\left(\hat{\mathbf{i}} \times \mathbf{e}_{32}\right)^{k}\right\}
\end{aligned}
$$

$$
\begin{aligned}
& \frac{\partial^{3} \tau}{\partial \mathbf{x}_{23}^{i}} \partial \mathbf{x}_{23}^{j} \partial \mathbf{x}_{23}^{k} \\
&= \frac{1}{R_{b}^{3}}\left\{\left[2 \left(F_{2} \mathbf{v}_{1}^{i} \mathbf{v}_{1}^{j}-r_{23} \frac{\partial F_{2}}{\partial \mathbf{x}_{23}^{i}} r_{21} \frac{\partial F_{2}}{\partial \mathbf{x}_{21}^{j}}\right.\right.\right. \\
&\left.-r_{23} \frac{\partial F_{2}}{\partial \mathbf{x}_{23}^{j}} r_{21} \frac{\partial F_{2}}{\partial \mathbf{x}_{21}^{i}}+F_{2}\left(\mathbf{e}_{21}^{i} \mathbf{e}_{21}^{j}-\delta^{i j}\right)\right) \\
&\left.+G_{2}^{2} r_{23}^{2} \frac{\partial^{2} F_{2}}{\partial \mathbf{x}_{23}^{i} \partial \mathbf{x}_{23}^{j}}\right] \mathbf{u}_{2}^{k} \\
&\left.+\mathbf{w}_{22}^{i}\left(\hat{\mathbf{j}} \times \mathbf{e}_{21}\right)^{k}+\mathbf{w}_{22}^{j}\left(\hat{\mathbf{i}} \times \mathbf{e}_{21}\right)^{k}\right\} \quad(\mathrm{a} 32)
\end{aligned}
$$$$
\frac{\partial^{3} \tau}{\partial \mathbf{x}_{32}^{i} \partial \mathbf{x}_{32}^{j} \partial \mathbf{x}_{32}^{k}}
$$$$
=\frac{-1}{R_{c}^{3}}\left\{\left[2 \left(F_{3} \mathbf{v}_{4}^{i} \mathbf{v}_{4}^{j}-r_{34} \frac{\partial F_{3}}{\partial \mathbf{x}_{34}^{i}} r_{32} \frac{\partial F_{3}}{\partial \mathbf{x}_{32}^{j}}\right.\right.\right.
$$$$
\left.-r_{34} \frac{\partial F_{3}}{\partial \mathbf{x}_{34}^{j}} r_{32} \frac{\partial F_{3}}{\partial \mathbf{x}_{32}^{i}}+F_{3}\left(\mathbf{e}_{34}^{i} \mathbf{e}_{34}^{j}-\delta^{i j}\right)\right)
$$$$
\left.+G_{3}^{2} r_{32}^{2} \frac{\partial^{2} F_{3}}{\partial \mathbf{x}_{32}^{i} \partial \mathbf{x}_{32}^{j}}\right] \mathbf{u}_{3}^{k}
$$$$
\left.+\mathbf{w}_{33}^{i}\left(\mathbf{e}_{34} \times \hat{\mathbf{j}}\right)^{k}+\mathbf{w}_{33}^{j}\left(\mathbf{e}_{34} \times \hat{\mathbf{i}}\right)^{k}\right\}
$$

$$
\begin{aligned}
& \frac{\partial^{3} \tau}{\partial \mathbf{x}_{23}^{i} \partial \mathbf{x}_{21}^{j} \partial \mathbf{x}_{21}^{k}} \\
& =\frac{-1}{R_{b} R_{a}^{2}}\left\{\left[\left(\mathbf{v}_{1}^{i}+\mathbf{w}_{21}^{i}\right) \mathbf{v}_{2}^{j}+2\left(G_{2}\left(\hat{\mathbf{i}} \times \mathbf{u}_{2}\right)^{j}\right.\right.\right. \\
& \left.\left.-F_{2}\left(\mathbf{e}_{21}^{i} \mathbf{e}_{21}^{j}-\delta^{i j}\right)-\mathbf{e}_{21}^{j} r_{21} \frac{\partial F_{2}}{\partial \mathbf{x}_{21}^{i}}\right)\right] \mathbf{u}_{2}^{k} \\
& +G_{2}(\hat{\mathbf{i}} \times \hat{\mathbf{j}})^{k}+\mathbf{w}_{21}^{i}\left(\mathbf{e}_{23} \times \hat{\mathbf{j}}\right)^{k} \\
& \left.+\mathbf{v}_{2}^{j}\left(\hat{\mathbf{i}} \times \mathbf{e}_{21}\right)^{k}\right\} \\
& \frac{\partial^{3} \boldsymbol{\tau}}{\partial \mathbf{x}_{32}^{i} \partial \mathbf{x}_{34}^{j} \partial \mathbf{x}_{34}^{k}} \\
& =\frac{1}{R_{c} R_{d}^{2}}\left\{\left[\left(\mathbf{v}_{4}^{i}+\mathbf{w}_{34}^{i}\right) \mathbf{v}_{3}^{j}+2\left(G_{3}\left(\mathbf{u}_{3} \times \hat{\mathbf{i}}\right)^{j}\right.\right.\right. \\
& \left.\left.-F_{3}\left(\mathbf{e}_{34}^{i} \mathbf{e}_{34}^{j}-\delta^{i j}\right)-\mathbf{e}_{34}^{j} r_{34} \frac{\partial F_{3}}{\partial \mathbf{x}_{34}^{i}}\right)\right] \mathbf{u}_{3}^{k} \\
& +G_{3}(\hat{\mathbf{j}} \times \hat{\mathbf{i}})^{k}+\mathbf{w}_{34}^{i}\left(\hat{\mathbf{j}} \times \mathbf{e}_{32}\right)^{k} \\
& \left.+\mathbf{v}_{3}^{j}\left(\mathbf{e}_{34} \times \hat{\mathbf{i}}\right)^{k}\right\} \\
& \frac{\partial^{3} \boldsymbol{\tau}}{\partial \mathbf{x}_{23}^{i} \partial \mathbf{x}_{23}^{j} \partial \mathbf{x}_{21}^{k}} \\
& =\frac{-1}{R_{b}^{2} R_{a}}\left\{\left[\mathbf{v}_{1}^{i} \mathbf{v}_{1}^{j}+\mathbf{w}_{21}^{i} \mathbf{w}_{21}^{j}\right.\right. \\
& \left.+2\left(\mathbf{e}_{21}^{i} \mathbf{e}_{21}^{j}-\delta^{i j}\right)-G_{2}^{2}\left(2 \mathbf{e}_{23}^{i} \mathbf{e}_{23}^{j}-\delta^{i j}\right)\right] \mathbf{u}_{2}^{k} \\
& \left.+\mathbf{w}_{21}^{i}\left(\hat{\mathbf{j}} \times \mathbf{e}_{21}\right)^{k}+\mathbf{w}_{21}^{j}\left(\hat{\mathbf{i}} \times \mathbf{e}_{21}\right)^{k}\right\} \\
& \frac{\partial^{3} \boldsymbol{\tau}}{\partial \mathbf{x}_{32}^{i} \partial \mathbf{x}_{32}^{j} \partial \mathbf{x}_{34}^{k}} \\
& =\frac{1}{R_{c}^{2} R_{d}}\left\{\left[\mathbf{v}_{4}^{i} \mathbf{v}_{4}^{j}+\mathbf{w}_{34}^{i} \mathbf{w}_{34}^{j}\right.\right. \\
& \left.+2\left(\mathbf{e}_{34}^{i} \mathbf{e}_{34}^{j}-\delta^{i j}\right)-G_{3}^{2}\left(2 \mathbf{e}_{32}^{i} \mathbf{e}_{32}^{j}-\delta^{i j}\right)\right] \mathbf{u}_{3}^{k} \\
& \left.+\mathbf{w}_{34}^{i}\left(\mathbf{e}_{34} \times \hat{\mathbf{j}}\right)^{k}+\mathbf{w}_{34}^{j}\left(\mathbf{e}_{34} \times \hat{\mathbf{i}}\right)^{k}\right\}
\end{aligned}
$$

\section{References}

1. Lee, S.-H.; Krimm, S. Biopolymers 1998, 46, 283.

2. Palmo, K.; Mirkin, N. G.; Krimm, S. J Phys Chem 1998, A102, 6448.

3. MacKerell, A. D., Jr.; Bashford, D.; Bellott, M.; Dunbrack, R. L., Jr.; Evanseck, J. D.; Field, M. J.; Fischer, S.; Gao, J.; Guo, H.; Ha, S.; Joseph-McCarthy, D.; Kuchnir, L.; Kucz- 


\section{LEE, PALMO, AND KRIMM}

era, K.; Lau, F. T. K.; Mattos, C.; Michnick, S.; Ngo, T.; Nguyen, D. T.; Prodhom, B.; Reiher, W. E., III; Roux, B.; Schlenkrich, M.; Smith, J. C.; Stote, R.; Straub, J.; Watanabe, M.; Wiórkiewicz-Kuczera, J.; Yin, D.; Karplus, M. J Phys Chem 1998, B102, 3586.

4. Maple, J. R.; Hwang, M.-J.; Jalkanen, K. J.; Stockfisch, T. P.; Hagler, A. T. J Comput Chem 1998, 19, 430.

5. Halgren, T. A. J Comput Chem 1996, 17, 553.

6. Nevins, N.; Chen, K.; Allinger, N. L. J Comput Chem 1996, 17,669 .

7. Cornell, W. D.; Cieplak, P.; Bayly, C. I.; Gould, I. R.; Merz, K. M., Jr.; Ferguson, D. M.; Spellmeyer, D. C.; Fox, T.; Caldwell, J. W.; Kollman, P. A. J Am Chem Soc 1995, 117, 5179.

8. Wilson, E. B.; Decius, J. C.; Cross, P. C. In Molecular Vibrations; McGraw-Hill: New York, 1955.

9. Schlick, T. J Comput Chem 1989, 10, 951.

10. Noid, D. W.; Sumpter, B. G.; Wunderlich, B.; Pfeffer, G. A. J Comput Chem 1990, 11, 236.

11. Swope, W. C.; Ferguson, D. M. J Comput Chem 1992, 13, 585.

12. Blondel, A.; Karplus, M. J Comput Chem 1996, 17, 1132.

13. Tuzun, R. E.; Noid, D. W.; Sumpter, B. G. Macromol Theory Simul 1995, 4, 909.
14. Miller, K. J.; Hinde, R. J.; Anderson, J. J Comput Chem 1989, $10,63$.

15. Miller, K. J. J Comput Chem 1990, 11, 336.

16. Lee, S.-H. Ph.D. thesis, University of Michigan (1997).

17. Tuzun, R. E.; Noid, D. W.; Sumpter, B. G. J Comput Chem 1997, 18, 1804

18. Plíva, J. Coll Czech Chem Commun 1958, 23, 777.

19. Dennison, D. M.; Uhlenbeck, G. E. Phys Rev 1932, 41, 313.

20. Califano, S. In Vibrational States; Wiley: New York, 1976.

21. Mills, I. M. Chem Phys Lett 1969, 3, 267.

22. Palmo, K.; Pietilä, L.-O.; Krimm, S. J Comput Chem 1992, 13, 1142.

23. Rush, D. J.; Wiberg, K. B. J Phys Chem 1997, A101, 3143.

24. Császár, A. G.; Allen, W. D.; Schaefer, H. F., III J Chem Phys 1998, 108, 9751.

25. Dennison, D. M.; Hardy, J. D. Phys Rev 1932, 39, 938.

26. Papousek, D.; Stone, J. M. R.; Spirko, V. J Mol Spectrosc 1973, 48, 17.

27. Spirko, V. J Mol Spectrosc 1983, 101, 30.

28. Koput, J. Chem Phys 1990, 148, 299.

29. Durig, J. R.; Flanagan, M. J.; Kalasinsky, V. F. J Chem Phys 1977, 66, 2775.

30. Sun, H.; Rigby, D. Spectrochim Acta 1997, A53, 1301. 